\title{
Cosmic Censorship for Gowdy Spacetimes
}

\author{
Hans Ringström \\ Department of Mathematics, Royal Institute of Technology \\ S-100 44 Stockholm, Sweden \\ email: hansr@kth.se \\ http://www.math.kth.se/ hansr/
}

Accepted on 29 March 2010

Published on 13 April 2010

\begin{abstract}
Due to the complexity of Einstein's equations, it is often natural to study a question of interest in the framework of a restricted class of solutions. One way to impose a restriction is to consider solutions satisfying a given symmetry condition. There are many possible choices, but the present article is concerned with one particular choice, which we shall refer to as Gowdy symmetry. We begin by explaining the origin and meaning of this symmetry type, which has been used as a simplifying assumption in various contexts, some of which we shall mention. Nevertheless, the subject of interest here is strong cosmic censorship. Consequently, after having described what the Gowdy class of spacetimes is, we describe, as seen from the perspective of a mathematician, what is meant by strong cosmic censorship. The existing results on cosmic censorship are based on a detailed analysis of the asymptotic behavior of solutions. This analysis is in part motivated by conjectures, such as the BKL conjecture, which we shall therefore briefly describe. However, the emphasis of the article is on the mathematical analysis of the asymptotics, due to its central importance in the proof and in the hope that it might be of relevance more generally. The article ends with a description of the results that have been obtained concerning strong cosmic censorship in the class of Gowdy spacetimes.
\end{abstract}

This review is licensed under a Creative Commons Attribution-Non-Commercial-NoDerivs 3.0 Germany License. http://creativecommons.org/licenses/by-nc-nd/3.0/de/ 


\section{Imprint / Terms of Use}

Living Reviews in Relativity is a peer reviewed open access journal published by the Max Planck Institute for Gravitational Physics, Am Mühlenberg 1, 14476 Potsdam, Germany. ISSN 1433-8351.

This review is licensed under a Creative Commons Attribution-Non-Commercial-NoDerivs 3.0 Germany License: http://creativecommons.org/licenses/by-nc-nd/3.0/de/

Because a Living Reviews article can evolve over time, we recommend to cite the article as follows:

Hans Ringström,

"Cosmic Censorship for Gowdy Spacetimes",

Living Rev. Relativity, 13, (2010), 2. [Online Article]: cited [<date $>$ ], http://www.livingreviews.org/lrr-2010-2

The date given as $<$ date $>$ then uniquely identifies the version of the article you are referring to.

\section{Article Revisions}

Living Reviews supports two ways of keeping its articles up-to-date:

Fast-track revision A fast-track revision provides the author with the opportunity to add short notices of current research results, trends and developments, or important publications to the article. A fast-track revision is refereed by the responsible subject editor. If an article has undergone a fast-track revision, a summary of changes will be listed here.

Major update A major update will include substantial changes and additions and is subject to full external refereeing. It is published with a new publication number.

For detailed documentation of an article's evolution, please refer to the history document of the article's online version at http://www. livingreviews.org/lrr-2010-2. 


\section{Contents}

1 Introduction and Outline $r$

1.1 Outline, basic material . . . . . . . . . . . . . . . . . . 7

1.2 Outline, the asymptotics in the direction towards the singularity . . . . . . . . 8

1.3 Outline, the expanding direction . . . . . . . . . . . . . . . . . 8

1.4 Strong cosmic censorship . . . . . . . . . . . . . . . . . . . 9

2 Gowdy Spacetimes $\quad 10$

2.1 Symmetry in cosmology . . . . . . . . . . . . . . . . . . . . . . 10

2.1.1 Symmetry via the Lie algebra . . . . . . . . . . . . . . . . . . . . 10

2.1.2 Symmetry via Lie group actions on the spacetime . . . . . . . . . . . 10

2.1.3 Symmetry via the initial value formulation . . . . . . . . . . . . . . 10

2.1.4 Cosmological symmetry hierarchy . . . . . . . . . . . . . . . . 10

2.1.5 Limitations, different perspectives . . . . . . . . . . . . . . . . . . . . . . . . . . . . . . .

2.1 .6 Present status, hierarchy . . . . . . . . . . . . . . . . 11

2.2 Definition of the Gowdy class . . . . . . . . . . . . . . . . . . . . . . . . . . . . . . . . . . . . . .

2.2.1 Twist constants, two-surface orthogonality . . . . . . . . . . . . 11

2.2.2 Essential characterizing conditions . . . . . . . . . . . . . . . 12

2.2.3 Technical definition . . . . . . . . . . ........ 12

2.3 Coordinate systems . . . . . . . . . . . . . . . . . . . . 12

2.3.1 Coordinate systems, $T^{3}$-Gowdy . . . . . . . . . . . . . . . . . 12

2.3.2 Working definition, $T^{3}$-Gowdy . . . . . . . . . . . . . . . . . . 12

2.3.3 Coordinate system, $S^{3}$ and $S^{2} \times S^{1} \ldots \ldots \ldots \ldots$

2.4 The polarized subcase . . . . . . . . . . . . . . . . 13

3 Foliations 14

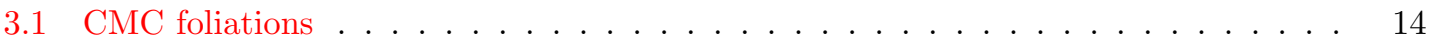

3.2 Areal foliation . . . . . . . . . . . . . . . . . . . . . . . . . . . . . . . . . . . . . . . .

3.3 Existence of foliations, related symmetry classes . . . . . . . . . . . . . . . 14

3.4 Prescribed mean curvature . . . . . . . . . . . . . . . . . . . . . . 14

4 Strong Cosmic Censorship $\quad 16$

4.1 The initial value problem . . . . . . . . . . . . . . . . . . . 16

4.1 The vacuum equations . . . . . . . . . . . . . . . . . . . . . . . . . . .

4.1 .2 Formulation, intuition . . . . . . . . . . . . . . . . . . . 17

4.1.3 Formulation, formal definition . . . . . . . . . . . . . . . . 17

4.1.4 Existence of a development . . . . . . . . . . . . . . . . 18

4.1.5 Existence of a maximal globally-hyperbolic development . . . . . . . . 18

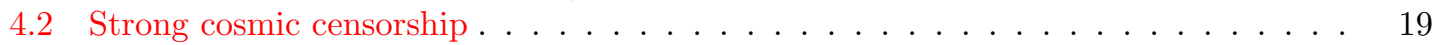

4.2 .1 Genericity . . . . . . . . . . . . . . . . . . . . . . . . . . . . . . . . . .

4.2 .2 Inextendibility . . . . . . . . . . . . . . . . . . . . . . . . . . . . . . . . . .

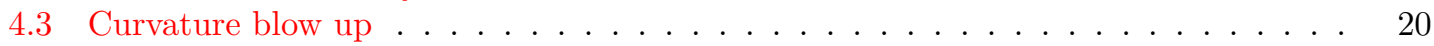

4.4 Pathological examples in the case of Gowdy . . . . . . . . . . . . . . . . . 20

5 BKL, Fuchsian Methods and Asymptotic Expansions 22

5.1 The BKL picture . . . . . . . . . . . . . . . . . . . . . . . . 22

5.2 Asymptotic expansions, Fuchsian methods . . . . . . . . . . . . . . . . . . . . . . . . . . . . . . . . . . . .

5.2.1 From solutions to asymptotics . . . . . . . . . . . . . . . 23

5.2 .2 From asymptotics to solutions . . . . . . . . . . . . . . . . . . . . . . . . . . . .

5.2 .3 Overview of results . . . . . . . . . . . . . . . . 24 
6 Equations $\quad 25$

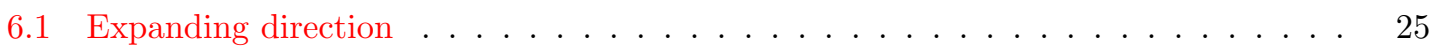

6.2 The direction towards the singularity . . . . . . . . . . . . . . . . 25

6.3 Wave-map structure . . . . . . . . . . . . . . . . . . . . . 25

6.3 .1 Representations of hyperbolic space . . . . . . . . . . . . . . 26

6.4 Conserved quantities, kinetic energy density . . . . . . . . . . . . . . . . 26

7 Singularity, Polarized Case $\quad 28$

7.1 Equations, polarized $T^{3}$-Gowdy . . . . . . . . . . . . . . . . . . . . . . . . . . . . . . . .

7.2 Associated Velocity Term Dominated system . . . . . . . . . . . . . . . . 28

7.3 Asymptotics of the solution to the polarized $T^{3}$-Gowdy equations . . . . . . . . . 28

7.4 Curvature blow up, polarized $T^{3}$-case . . . . . . . . . . . . . . . . . 29

7.5 Asymptotic velocity, polarized $T^{3}$-case . . . . . . . . . . . . . . . . . . 29

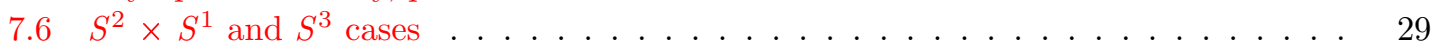

8 Asymptotic Expansions Using Fuchsian Methods, General $T^{3}$-Case 30

8.1 Geometric interpretation of $v_{a} \ldots \ldots \ldots \ldots$. . . . . . . . . . . . . . . . . . . . . . . . . .

8.2 Restriction on the velocity . . . . . . . . . . . . . . . . . . 30

8.3 Geodesic loop . . . . . . . . . . . . . . . . . . . . . 30

8.4 Existence of expansions using Fuchsian methods, $T^{3}$-case . . . . . . . . . . . . 31

8.5 Existence of expansions using Fuchsian methods, $S^{2} \times S^{1}$ and $S^{3}$ cases . . . . . 31

9 Spikes $\quad 32$

9.1 Inversion . . . . . . . . . . . . . . . . . . . . . . . . . . 32

9.2 Gowdy to Ernst transformation . . . . . . . . . . . . . . . . . . . . . . . . . . . . . . . . . . . . .

9.3 False spikes . . . . . . . . . . . . . . . . . . . . . 32

9.3 .1 True spikes . . . . . . . . . . . . . . . . . . 35

9.4 High velocity spikes . . . . . . . . . . . . . . . . . . . . . 36

10 Asymptotic Velocity, General $T^{3}$-Gowdy $\quad 37$

10.1 Existence of an asymptotic velocity . . . . . . . . . . . . . . . 37

10.2 Relevance of the asymptotic velocity to the issue of curvature blow up . . . . . . . 37

10.3 Interpretation of the asymptotic velocity as a rate of convergence to the boundary in hyperbolic space . . . . . . . . . . . . . . . . . . . . . . . . . . . . . 37

10.4 Two dimensional version of the asymptotic velocity . . . . . . . . . . . . . . . . . . . . . . . . . . . .

10.5 Dominant contribution to the asymptotic velocity . . . . . . . . . . . . 38

10.6 Value of the asymptotic velocity as a criterion for the existence of expansions . . . 38

11 The Generic Set, General T Th-Gowdy 40

11.1 Nondegenerate true spikes . . . . . . . . . . . . . . . . . . . . . 40

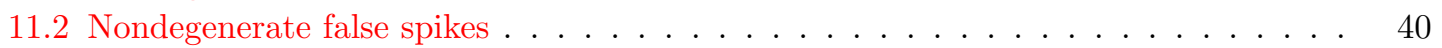

11.3 The generic set, definition . . . . . . . . . . . . . . . . . . . . . . . . . . . . . . . . . . . . . . . . . .

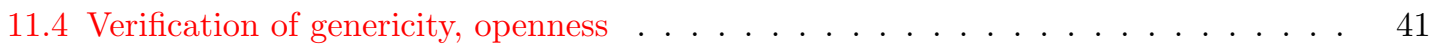

11.5 Verification of genericity, density . . . . . . . . . . . . . . . . . . 41

12 Expanding Direction, Polarized Gowdy 42

12.1 Asymptotic behavior . . . . . . . . . . . . . . . . . . . . . 42

12.2 Comparison with spatially homogeneous solutions . . . . . . . . . . . . . 43 
13 Expanding Direction, The General Case 44

13.1 Energy decay . . . . . . . . . . . . . . . . . . . . . . . . . . . . . . . . . . . . . . . . . . . . .

13.2 Proof of decay of the energy . . . . . . . . . . . . . . . . . . . . . . . . . . . . . . . . . . . . . . . . .

13.2.1 Toy model . . . . . . . . . . . . . . . . . . . . . . . . . . . . . . . . . . . . . . . . 45

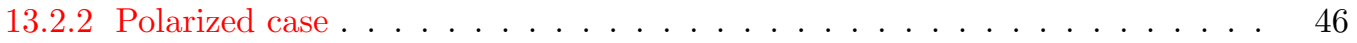

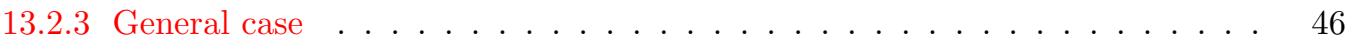

13.3 Asymptotic ODE behavior . . . . . . . . . . . . . . . . . . . . . 46

13.3.1 Conserved quantities . . . . . . . . . . . . . . . . . . . 47

13.3.2 Interpreting the conserved quantities as ODEs for the averages . . . . . 47

13.4 Geometric interpretation of the asymptotics . . . . . . . . . . . . . . . . . . 49

13.5 Concluding remarks . . . . . . . . . . . . . . . . . . . . 50

13.6 Geodesic completeness . . . . . . . . . . . . . . . . . . . 50

14 Strong Cosmic Censorship in Gowdy Spacetimes $\quad 51$

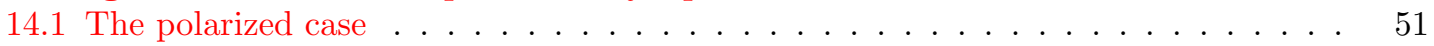

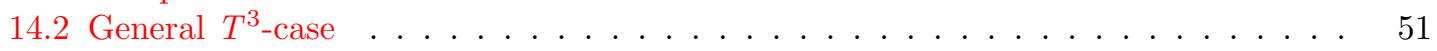

15 Acknowledgments $\quad 53$

$\begin{array}{ll}\text { References } & 54\end{array}$ 



\section{Introduction and Outline}

Gowdy spacetimes have been used as a toy model in the context of, e.g., gravitational waves, quantum gravity, numerical relativity and mathematical cosmology. However, here we shall only be concerned with the question of strong cosmic censorship. In other words, we are interested in a mathematical problem. Nevertheless, numerical relativity has played an important role in the development of the subject, and as a consequence, we shall mention some of the key numerical observations.

\subsection{Outline, basic material}

Definition of the Gowdy class. Since the present article is concerned with cosmic censorship in Gowdy spacetimes, a natural starting point is to define the Gowdy class. This is the subject of Section 2. However, in order to obtain a good understanding, it is of interest to put this symmetry class into perspective. Therefore, in Section 2.1, we discuss the role of symmetry in cosmology. In particular, we mention different ways of imposing symmetry and describe the place the Gowdy spacetimes occupy in the symmetry hierarchy. In Section 2.2, we then define the Gowdy spacetimes. The essential condition is that there be a two-dimensional isometry group with two-dimensional spacelike orbits. However, Gowdy makes some additional restrictions, which we explain in Section 2.2.1. We end the section by defining an important subclass called polarized Gowdy; see Section 2.4.

The existence of foliations. After the Gowdy class has been defined, a natural first question to ask is if there are preferred foliations. For example, is there a CMC foliation, and, if so, does it cover the maximal globally-hyperbolic development (MGHD)? We address such questions in Section 3.

Formulation of the strong cosmic-censorship conjecture. In Section 4, we turn to the formulation of the strong cosmic-censorship conjecture. We shall here phrase it in terms of the initial value problem. Therefore, in Section 4.1, we define the initial value problem for Einstein's equations. First, we give an intuitive motivation for some aspects of the formulation. We then provide a formal definition. After having phrased the problem, we mention the standard results concerning the existence of developments. The emphasis is on the existence of the MGHD. In Section 4.2, we then state the strong cosmic censorship conjecture. Two words that require a detailed definition occur in the formulation: generic and inextendible. There are several possible technical definitions of these concepts, and we provide some examples. We end the section by formulating a related conjecture concerning curvature blow up in Section 4.3 and by mentioning some pathologies that can occur in Gowdy in Section 4.4.

The BKL conjecture. The results that exist concerning strong cosmic censorship in Gowdy spacetimes have been obtained through a detailed analysis of the asymptotic behavior of solutions. One point of view that has played an important role in the analysis is the circle of ideas often referred to as the "BKL conjecture" (after Belinskii, Khalatnikov, and Lifshitz). For this reason, in Section 5, we give a brief description of these ideas as well as some recent developments. A related topic is that of asymptotic expansions, which we discuss in Section 5.2. We also describe the Fuchsian methods that can be used to prove that there are solutions with a prescribed type of asymptotic behavior.

The equations. In Section 6, we write down Einstein's equations in terms of the components of a $T^{3}$-Gowdy metric. It is important to note that the essential equations have the structure of a wave-map with hyperbolic space as a target. We describe this structure and mention some of its consequences. 


\subsection{Outline, the asymptotics in the direction towards the singularity}

Asymptotic behavior in the direction of the singularity in the polarized case. The two Gowdy cases in which results concerning strong cosmic censorship exist are the polarized case and the general $T^{3}$-case. In Section 7, we focus on the polarized case. In particular, on finding asymptotic expansions of the metric components in the direction towards the singularity. One function appearing in the expansions has a special importance. From the wave-map point of view, it has a natural interpretation as the rate at which the solution tends to the boundary of hyperbolic space; see Section 7.4. As a consequence, it is referred to as the asymptotic velocity. Beyond having a natural interpretation, the asymptotic velocity has an additional important property. In fact, it can be used as a criterion for curvature blow up along causal curves going into the singularity; see Section 7.5 .

Existence of solutions with specified asymptotics, Fuchsian methods. Due to the central importance of the asymptotic expansions in the polarized case, it is of interest to obtain expansions in the general $T^{3}$-case. One way to proceed is to try to construct solutions with prescribed asymptotics. This is the subject of Section 8. Again, it is possible to define the concept of an asymptotic velocity. It has the same geometric interpretation and importance as in the polarized case. The results on existence of expansions depend on a restriction of the asymptotic velocity. We describe the results and motivate the restriction.

Spikes. The numerical studies indicate that for most spatial points, the asymptotic expansions presented in Section 8 constitute a good description of the asymptotics. However, they also indicate that there are spatial points where the behavior is very different. Due to the visual impression of plots of the solutions in the neighborhood of the exceptional points, the corresponding features have been referred to as "spikes". In Section 9, we describe analytic constructions of solutions with spikes.

Existence of an asymptotic velocity in the general $\boldsymbol{T}^{\mathbf{3}}$-Gowdy case. The analysis in the polarized case and the construction of solutions with prescribed asymptotics indicate the importance of the asymptotic velocity. Consequently, it is of interest to prove that the asymptotic velocity exists in general. This is the subject of Section 10. We also demonstrate that the asymptotic velocity can be viewed as a two-dimensional object in the disc model. Finally, we illustrate that it can be used as a criterion for the existence of expansions.

Definition of the generic set in the general $T^{\mathbf{3}}$-Gowdy case. As a preparation for the formulation of the theorem verifying that strong cosmic censorship holds in $T^{3}$-Gowdy, we define the generic set of initial data in Section 11.

\subsection{Outline, the expanding direction}

The asymptotic behavior in the expanding direction of polarized Gowdy. Only in the case of $T^{3}$-topology is there an expanding direction. Consequently, it is only necessary to discuss the general $T^{3}$-case. However, there are some results of interest, which are only known in the polarized case. Consequently, we devote Section 12 to a discussion of it. It is of particular interest to note that the spatial variation of solutions dies out in the sense that the difference between the solution and its average converges to zero. On the other hand, with respect to other measures, the solution does not tend to spatial homogeneity.

The asymptotic behavior in the expanding direction of general $T^{\mathbf{3}}$-Gowdy. In the general case, less detailed information is available. However, a clear picture of the asymptotics exists and is described in Section 13. The first step of the analysis consists of proving that a naturally defined energy converges to zero at a specific rate. This leads to the conclusion that the distance from the solution to its average converges to zero. In order to analyze the asymptotics of solutions, it is convenient to note that there are conserved quantities. When viewed in the right way, these conserved quantities can be reinterpreted as ODEs for the averages, and this leads to

Living Reviews in Relativity

http: //www. livingreviews.org/lrr-2010-2 
detailed information concerning their asymptotics. Finally, results concerning the decay of the sup norm of derivatives is derived. Such estimates are useful in order to prove future causal geodesic completeness.

\subsection{Strong cosmic censorship}

Strong cosmic censorship. Finally, in Section 14, we phrase the existing results on strong cosmic censorship in the class of Gowdy spacetimes. So far, the results are restricted to the polarized case and the general $T^{3}$-case. 


\section{Gowdy Spacetimes}

In order to put the Gowdy class of spacetimes into context, it is natural to start by discussing the role of symmetry in general relativity. We shall not discuss it here in all generality, but will restrict our attention to the four-dimensional cosmological case.

\subsection{Symmetry in cosmology}

By a cosmological spacetime, we mean one that is foliated by compact spacelike hypersurfaces. Moreover, we shall, most of the time, tacitly assume the spacelike hypersurfaces to be Cauchy hypersurfaces; see Definition 2. Let us mention a few different ways of imposing symmetry conditions.

\subsubsection{Symmetry via the Lie algebra}

In the physics literature, it is quite common to phrase the demands in terms of the Lie algebra of Killing vector fields on the spacetime under consideration.

\subsubsection{Symmetry via Lie group actions on the spacetime}

Another possibility is to demand that there be a Lie group acting smoothly and effectively by isometries on the spacetime. Recall that a Lie group action $G \times M \rightarrow M$ is effective if $g p=p$ for all $p \in M$ implies $g=e$.

\subsubsection{Symmetry via the initial value formulation}

A third option is provided by the formulation of Einstein's equations of general relativity as an initial value problem. We shall give a more complete presentation of the initial value formulation in Section 4.1. However, let us briefly recall the main ingredients here. In the cosmological case, the initial data consist of a three-dimensional compact manifold $\Sigma$ on which a Riemannian metric, a symmetric covariant two-tensor and suitable matter fields are specified. Assuming the matter model to be of an appropriate type, there is a unique MGHD of the initial data; see Theorem 2. One way to impose symmetries is to demand that there be a Lie group acting smoothly and effectively by isometries on the initial data. In order for this perspective to be of any interest, such a Lie group action should give rise to a smooth effective Lie group action, acting by isometries, on the MGHD. That this is the case can be seen by the argument presented in [70, pp. 176-177]; see also $[17,18]$.

\subsubsection{Cosmological symmetry hierarchy}

In the study of the initial value problem, it is of interest to analyze what combinations of compact Lie groups and compact three-dimensional manifolds $\Sigma$ are such that there is a smooth and effective Lie group action of $G$ on $\Sigma$. It turns out that there are quite a limited number of possibilities. The introduction of [17] contains a list. Readers interested in the underlying mathematics are referred to [59]. Given a specific topic of interest, such as, the strong cosmic-censorship conjecture, this list yields a hierarchy of classes of spacetimes in which one can study it in a simplified setting.

\subsubsection{Limitations, different perspectives}

It should be noted that requiring the existence of an effective Lie group action of the type described above excludes large classes of cosmological spacetimes that are, in some respects, of a high degree of symmetry. Most spatially locally-homogeneous cosmological models are excluded. A more

Living Reviews in Relativity

http: //www. livingreviews.org/Irr-2010-2 
natural perspective to take would perhaps be to demand that there be an appropriate Lie group action on the universal covering space. Yet another perspective is provided by [87]. The central assumption of [87] is the existence of two commuting local Killing vectors, and a larger class of spatial topologies is thereby permitted, see also [67].

\subsubsection{Present status, hierarchy}

In the case of cosmology, the assumption of spatial local homogeneity is a natural starting point. However, in this setting, the issue of strong cosmic censorship is quite well understood. Note that this claim rests on our particular definition of a cosmological spacetime. In fact, most Bianchi class B solutions are excluded by the condition that they should admit spatially compact quotients. On the other hand, it should be pointed out that there are many fundamental problems that have not been sorted out even in the spatially homogeneous setting; the detailed asymptotics of Bianchi IX and the question of whether particle horizons form in Bianchi VIII and IX or not are but two examples, see, for example, $[72,73,45,46]$ and references cited therein for partial results concerning the asymptotics and [44] for a discussion of the issue of particle horizons.

When proceeding beyond spatial homogeneity, the natural next step is to consider the case of a two-dimensional isometry group. This leads us to the Gowdy class of spacetimes.

\subsection{Definition of the Gowdy class}

Spacetimes admitting two-dimensional isometry groups had been studied prior to [39]. However, the considerations had mainly been limited to the stationary axisymmetric case. To the best of our knowledge, Gowdy was the first one to systematically analyze the consequences of imposing the existence of a two-dimensional isometry group with spacelike orbits [39, 38, 37]. As a consequence, a subclass of this family now bears his name. The main objective of the work presented in [39] is to write down a convenient global form for metrics admitting this type of symmetry. However, in the course of the discussion, Gowdy introduces assumptions that exclude a large family of solutions admitting a two-dimensional isometry group. Let us introduce the terminology necessary for describing the discarded class.

\subsubsection{Twist constants, two-surface orthogonality}

In order to be able to describe the class excluded by Gowdy's assumptions, let us note some of the consequences of the essential symmetry assumption - that there is a compact and connected twodimensional Lie group acting effectively on the initial hypersurface. Since the initial hypersurface is a three-dimensional manifold, [59, Theorem 6, p. 453] implies that the Lie group has to be $T^{2}$; i.e., $U(1) \times U(1)$. In particular, there are initially two Killing fields that then extend to two spacelike Killing fields on the development. We shall denote them by $X_{i}, i=1,2$. As pointed out in [16, p. 101], the symmetry assumptions imply that the topology of the initial hypersurface has to be $T^{3}, S^{2} \times S^{1}, S^{3}$ or one of the Lens spaces. Since the Lens spaces have $S^{3}$ as a universal covering space, we shall consider them to be subsumed under that case. It is also possible to describe how the group acts on the manifold in some detail [16, p. 102]. In particular, there must be "axes" at which one of the Killing fields vanish for all topologies except $T^{3}$. Returning to the discarded class of solutions, let us define the functions

$$
c_{a}=\epsilon_{\alpha \beta \gamma \delta} X_{1}^{\alpha} X_{2}^{\beta} \nabla^{\gamma} X_{a}^{\delta},
$$

$a=1,2$. They are constant on the spacetime (this statement is true in the vacuum case [16, p. 103] but not necessarily in the presence of matter) and are referred to as the twist constants. Gowdy assumes them to vanish [39, p. 211]. Note, however, that he calls this specialization two-surface 
orthogonality, since it implies [16, Theorem 4.2, p. 117] that the group orbits are orthogonal to the vector field $\partial_{t}$, where $t$ is the areal time coordinate (i.e. the function that to each point of the spacetime associates the area of the group orbit containing the point). In the case of $S^{2} \times S^{1}$, $S^{3}$ and Lens space topology, the twist constants have to vanish due to the existence of the axes. However, in the case of $T^{3}$ topology, there is a class of solutions with nonvanishing twist constants called $T^{2}$-symmetric spacetimes. The behavior of solutions belonging to this class is much more complicated than that of those belonging to the Gowdy class of spacetimes.

\subsubsection{Essential characterizing conditions}

To conclude, the essential assumptions characterizing the Gowdy class are that a member of it should

- be a globally-hyperbolic cosmological spacetime,

- admit an effective action by isometries by a two-dimensional compact Lie group with spacelike orbits,

- be such that the twist constants vanish.

\subsubsection{Technical definition}

Even though the above list gives the central assumptions, there are some subtleties that have been sorted out in [16]. Thus, the formally inclined are recommended to use the assumptions of [16, Theorem 4.2, p. 117] and of [16, Theorem 6.1, p. 128-129] as a definition of Gowdy initial data. The Gowdy class of solutions is then defined as the MGHDs of Gowdy initial data.

\subsection{Coordinate systems}

In [16], special coordinate systems are constructed on part of the MGHD. Let us describe the different cases.

\subsubsection{Coordinate systems, $T^{3}$-Gowdy}

In the case of $T^{3}$-topology, there are coordinates such that the metric takes the form

$$
g_{T^{3}}=e^{2 B}\left(-d t^{2}+d \theta^{2}\right)+\ell t n_{a b}\left(d x^{a}+g^{a} d \theta\right)\left(d x^{b}+g^{b} d \theta\right) .
$$

Here $t \in(0, \infty), \theta, x^{a} \in[0,2 \pi] \bmod 2 \pi, B$ and $n_{a b}$ are functions of $t$ and $\theta, \ell>0$ is a constant, $\operatorname{det} n_{a b}=1$ and the $g^{a}$ are constants. This is a special case of the form of the metric given in [16, (4.9), p. 116]; see also [16, Theorem 4.2] and [16, (4.12), p. 117].

\subsubsection{Working definition, $T^{3}$-Gowdy}

The values of the constants $\ell$ and $g^{a}$ in Equation (1) are of no importance in practice. Consequently, $\ell$ can be taken to equal 1 and $g^{a}$ can be taken to equal 0 . In order to arrive at the form of the metric we shall actually be using, let us set $\ell=1$ and $g^{a}=0$. Furthermore, we define $x^{1}=: \sigma$, $x^{2}=: \delta, n_{11}=: e^{P}, n_{12}=e^{P} Q$, where we have used the fact that $n_{a b}$ are the components of a positive definite matrix. Since $n_{a b}$ is also a symmetric matrix with unit determinant, we obtain

$$
g_{T^{3}}=t^{-1 / 2} e^{\lambda / 2}\left(-d t^{2}+d \theta^{2}\right)+t\left[e^{P} d \sigma^{2}+2 e^{P} Q d \sigma d \delta+\left(e^{P} Q^{2}+e^{-P}\right) d \delta^{2}\right],
$$

where we have defined $\lambda$ by the relation $t^{-1 / 2} e^{\lambda / 2}=e^{2 B}$. An alternate definition of a $T^{3}$-Gowdy spacetime is a manifold of the form $I \times T^{3}$ with a metric of the form of Equation (2). Of course, some form of Einstein's equation should also be enforced. 


\subsubsection{Coordinate system, $S^{3}$ and $S^{2} \times S^{1}$}

In the case of $S^{3}$ and $S^{2} \times S^{1}$ topology, the metric can be written

$$
g_{S}=e^{2 B}\left(-d T^{2}+d \psi^{2}\right)+\lambda_{a b} d x^{a} d x^{b},
$$

where $x^{a} \in[0,2 \pi] \bmod 2 \pi, T \in(0, \pi), \psi \in[0, \pi]$, det $\lambda_{a b}=\ell \sin T \sin \psi$, where $\ell>0$ is a constant, and $B$ and $\lambda_{a b}$ are functions of $T$ and $\psi$. This is the form of the metric given in [16, Theorem 6.3 , p. 133], though it should again be pointed out that it is not claimed that the coordinates with respect to which the metric takes this form cover the entire MGHD.

\subsection{The polarized subcase}

The polarized Gowdy solutions constitute an interesting subclass. They are characterized by the additional condition that the Killing vectors be mutually orthogonal. In the polarized $T^{3}$-Gowdy case, the metric can be written

$$
g_{P}=t^{-1 / 2} e^{\lambda / 2}\left(-d t^{2}+d \theta^{2}\right)+t\left(e^{P} d \sigma^{2}+e^{-P} d \delta^{2}\right)
$$

i.e., it corresponds to setting $Q=0$ in Equation (2). In the case of $S^{3}$ and $S^{2} \times S^{1}$, the metric takes the form

$$
g_{S, P}=e^{2 a}\left(-d t^{2}+d \theta^{2}\right)+\sin \theta \sin t\left(e^{W} d x^{2}+e^{-W} d y^{2}\right)
$$

see Equations (5)-(7) of [50]. Clearly, the connection between the coordinates, the metric components and the topology is more complicated in the case of $S^{3}$ and $S^{2} \times S^{1}$ than in the case of $T^{3}$. We shall not go into a detailed discussion of these issues here, but rather refer the interested reader to [21] and [50] for a further discussion. A brief explanation of the origin of the name (in terms of polarizations of gravitational waves) as well as a different characterization is provided at the bottom of $[70$, p. 65]. 


\section{Foliations}

The components of a Gowdy metric, see Equations (2) and (3), are not explicit functions of the coordinates. However, imposing Einstein's equations leads to a system of nonlinear wave equations for the components. Consequently, it is useful to analyze the asymptotic behavior of the solutions to this system in order to be able to draw conclusions concerning the global geometry of the corresponding spacetimes. One natural first question to ask is if there are any preferred global foliations. Is there, e.g., a constant mean curvature (CMC) foliation?

\subsection{CMC foliations}

Note that CMC foliations are unique in cosmological vacuum spacetimes. In the case of vacuum $T^{3}$-Gowdy, there is a CMC foliation exhausting the interval $(-\infty, 0)$ [49]. However, in the case of $S^{2} \times S^{1}$ and $S^{3}$ topology, the only general statements concerning foliations are, as far as we are aware, the ones given in [16]. It is natural to conjecture that if there is a CMC Cauchy hypersurface in the $S^{2} \times S^{1}$ or $S^{3}$ case, there is a CMC foliation exhausting the interval $(-\infty, \infty)[66]$. However, to the best of our knowledge there are no results to this effect.

\subsection{Areal foliation}

In the case of $T^{3}$-Gowdy spacetimes, there is another natural foliation; considering Equation (2), it is clear that the area of the symmetry orbits are proportional to the time coordinate $t$. Consequently, such a time coordinate is referred to as an areal time coordinate. It is natural to ask if the areal time coordinate exhausts the interval $(0, \infty)$. That the answer is yes in the case of vacuum $T^{3}$-Gowdy was demonstrated by Moncrief [58]. Furthermore, he verified that the foliation covers the entire MGHD. However, since the starting point of the argument in [58] is a constant- $t$ hypersurface, it is of interest to note that the results of [16] yield the same conclusions starting with a general Cauchy hypersurface.

\subsection{Existence of foliations, related symmetry classes}

Let us, for the sake of completeness, mention some results concerning spacetimes satisfying related symmetry conditions, in particular $T^{2}$-symmetry; see Section 2.2. That the maximal globallyhyperbolic vacuum development of $T^{2}$-symmetric initial data is covered by areal coordinates is proven in [9]. The result states that the area of the symmetry orbits exhausts $(c, \infty)$ for some $c \geq 0$; whether $c=0$ or not is left open. However, this question has been addressed and resolved in [51] and [91], see also [85]. In the context of areal coordinates, there is a fundamental difference between the Gowdy case and the general $T^{2}$-symmetric case. In the Gowdy case, the areal time coordinate is such that the metric is conformal to the Minkowski metric in the $t \theta$-direction; see Equation (2). In the general $T^{2}$-symmetric case, this property is lost if one insists on an areal time coordinate [9]. Results on the existence of areal coordinates covering the MGHD in the case of solutions to the Einstein-Vlasov system with $T^{3}$-Gowdy symmetry are contained in [4], see also [5], which treats solutions to the Einstein-Vlasov system in the general $T^{2}$-symmetric case (the latter paper contains results concerning both areal and CMC foliations). Existence of a CMC foliation under the assumption of the existence of two local Killing vectors was demonstrated in [67], a paper, which generalizes, among other things, the results of [49].

\subsection{Prescribed mean curvature}

The above results concerning the existence of CMC foliations are based on the assumption of the existence of one CMC hypersurface. There are ways to circumvent this condition. By proving

Living Reviews in Relativity

http: //www. livingreviews.org/lrr-2010-2 
the existence of a suitable prescribed mean curvature (PMC) foliation, it is sometimes possible to construct barriers that imply the existence of one CMC hypersurface; see Section 3.6 of [69] and references cited therein for more details. 


\section{Strong Cosmic Censorship}

The idea of cosmic censorship goes back to the work of Roger Penrose; see [62] (reprinted in [64]) and [63]. It comes in two forms: weak and strong. The weak cosmic-censorship conjecture is concerned with isolated systems and essentially states that, generically, singularities should not be visible to an observer at infinity; see [90] for a more precise and extensive discussion. The strong cosmic censorship conjecture is a statement concerning the deterministic nature of the general theory of relativity. This is the form we are interested in here, and we shall phrase it in terms of initial data. Consequently, we need to formulate the initial value (or Cauchy) problem for Einstein's equations.

\subsection{The initial value problem}

How does one formulate an initial value problem for Einstein's equations? What should the initial data be? Is there uniqueness in any reasonable sense? These questions can be formulated in the presence of various matter fields, but let us, for the sake of simplicity, restrict our attention to the vacuum case here.

\subsubsection{The vacuum equations}

The vacuum equations are given by

$$
G=0,
$$

where

$$
G=\operatorname{Ric}-\frac{1}{2} S g
$$

is the Einstein tensor, Ric is the Ricci tensor and $S$ is the scalar curvature of a Lorentz manifold $(M, g)$. Clearly, Einstein's vacuum equations are equivalent to

$$
\text { Ric }=0 .
$$

Note that Ric can be thought of as a differential operator acting on the metric. A thorough discussion of the principal symbol of this operator, of the implications of the diffeomorphism invariance of the equations etc... can be found in [35], see also [15]. However, here we shall take a more pedestrian approach. Writing down Ric in local coordinates, we have

$$
R_{\mu \nu}=-\frac{1}{2} g^{\alpha \beta} \partial_{\alpha} \partial_{\beta} g_{\mu \nu}+\nabla_{(\mu} \Gamma_{\nu)}+g^{\alpha \beta} g^{\gamma \delta}\left[\Gamma_{\alpha \gamma \mu} \Gamma_{\beta \delta \nu}+\Gamma_{\alpha \gamma \mu} \Gamma_{\beta \nu \delta}+\Gamma_{\alpha \gamma \nu} \Gamma_{\beta \mu \delta}\right]
$$

where

$$
\Gamma_{\alpha \gamma \beta}=\frac{1}{2}\left(\partial_{\alpha} g_{\beta \gamma}+\partial_{\beta} g_{\alpha \gamma}-\partial_{\gamma} g_{\alpha \beta}\right), \quad \Gamma_{\alpha}=g_{\alpha \beta} g^{\mu \nu} \Gamma_{\mu \nu}^{\beta}, \quad \nabla_{\mu} \Gamma_{\nu}=\partial_{\mu} \Gamma_{\nu}-\Gamma_{\mu \nu}^{\alpha} \Gamma_{\alpha}
$$

and a parenthesis denotes symmetrization, i.e.,

$$
\nabla_{(\mu} \Gamma_{\nu)}=\frac{1}{2}\left(\nabla_{\mu} \Gamma_{\nu}+\nabla_{\nu} \Gamma_{\mu}\right)
$$

The notation $\nabla_{\mu} \Gamma_{\nu}$ is questionable due to the fact that $\Gamma_{\mu}$ are not the components of a covector. Nevertheless, we shall use it. The highest-order derivatives are contained in

$$
-\frac{1}{2} g^{\alpha \beta} \partial_{\alpha} \partial_{\beta} g_{\mu \nu}+\nabla_{(\mu} \Gamma_{\nu)}
$$




\subsubsection{Formulation, intuition}

If the second term in Equation (7) were not there, Equation (5) would, in local coordinates, be a nonlinear wave equation, and it would be straightforward to formulate an initial value problem. Furthermore, given a solution to Equation (5), it is possible to choose local coordinates such that $\Gamma_{\mu}$ vanishes and Equation (5) takes the form of a nonlinear wave equation. On the other hand, if Equation (5) were a nonlinear wave equation when expressed with respect to arbitrary coordinates, we would obtain uniqueness for the coordinate expression of the metric. This statement is incompatible with diffeomorphism invariance. Thus, even though Equation (5) in some respects can be viewed as a hyperbolic differential equation, the geometric aspect of the equation must not be forgotten.

Due to the above observations, it seems natural to expect the right PDE problem to formulate for Einstein's equations to be the initial value problem. Furthermore, it seems clear that this problem should be given a geometric formulation. Naively, one would expect it to be necessary to specify the metric and the first time derivative of the metric at the initial hypersurface. However, these quantities are not geometric. The induced metric and second fundamental form are, on the other hand, geometric quantities and they contain part of the information one would naively expect to need. Furthermore, they, in the end, turn out to constitute sufficient information. The question arises of what should be required of the initial hypersurface? Since we wish to avoid issues of consistency, we shall require the hypersurface to be such that it has no causal tangent vectors. In other words, we require that it be spacelike (this is, strictly speaking, not necessary; there are formulations in the null case as well; see, e.g., [65]).

\subsubsection{Formulation, formal definition}

The above discussion suggests the following. The initial data should, at the very minimum, consist of a manifold, say $\Sigma$ (which should be thought of as the initial hypersurface), a Riemannian metric on $\Sigma$, say $\rho$ (which should be thought of as the induced metric on the initial hypersurface), and a symmetric covariant two-tensor on $\Sigma$, say $\kappa$ (which should be thought of as the induced second fundamental form). On the other hand, if $(\Sigma, \rho, \kappa)$ is a hypersurface with induced metric and second fundamental form in a Lorentz manifold solving Equation (5), then $\rho$ and $\kappa$ have to satisfy the constraint equations:

$$
\begin{aligned}
r-\kappa_{i j} \kappa^{i j}+(\operatorname{tr} \kappa)^{2} & =0, \\
D^{j} \kappa_{j i}-D_{i}(\operatorname{tr} \kappa) & =0 .
\end{aligned}
$$

Here $r$ is the scalar curvature and $D$ is the Levi-Civita connection associated with $\rho$, and indices are raised and lowered with $\rho$. Equation (8), the Hamiltonian constraint, is equivalent to the equation $G(N, N)=0$, where $G$ is the Einstein tensor and $N$ is a normal vector to the hypersurface. Equation (9), the momentum constraint, is equivalent to the equation $G(N, X)=0$, where $X$ is any tangent vector to the hypersurface.

Definition 1 Initial data for Einstein's vacuum equations consist of a three-dimensional manifold $\Sigma$, a Riemannian metric $\rho$ and a covariant symmetric two-tensor $\kappa$ on $\Sigma$, both assumed to be smooth and to satisfy Equations (8)-(9). Given initial data, the initial value problem is that of finding a four-dimensional manifold $M$ with a Lorentz metric $g$ such that Equation (5) is satisfied, and an embedding $i: \Sigma \rightarrow M$ such that $i^{*} g=\rho$ and that if $k$ is the second fundamental form of $i(\Sigma)$, then $i^{*} k=\kappa$. Such a Lorentz manifold $(M, g)$ is called a development of the data. Furthermore, if $i(\Sigma)$ is a Cauchy hypersurface in $(M, g)$, then $(M, g)$ is referred to as a globally-hyperbolic development of the initial data. In both cases, the existence of an embedding $i$ is tacit.

Since the concepts Cauchy hypersurface and globally hyperbolic are referred to above, and will be of some importance below, let us recall how they are defined. 
Definition 2 Let $(M, g)$ be a Lorentz manifold. A subset $\Sigma$ of $M$ is said to be a Cauchy hypersurface if it is intersected exactly once by every inextendible timelike curve. A Lorentz manifold that admits a Cauchy hypersurface is said to be globally hyperbolic.

Remark. Two basic examples of Cauchy hypersurfaces are the $t=$ const. hypersurfaces in Minkowski space and the hypersurfaces of spatial homogeneity in Robertson-Walker spacetimes. The reader interested in the basic properties of globally-hyperbolic Lorentz manifolds and Cauchy hypersurfaces is referred to [60], see also [82] and references cited therein. Cauchy hypersurfaces need neither be smooth nor spacelike, but we shall tacitly assume them to be both. The reason the concept of a Cauchy hypersurface is of such central importance is that it is the natural type of surface on which to specify initial data.

\subsubsection{Existence of a development}

We are now in a position to ask: given initial data to Einstein's vacuum equations, is there a development? The answer to this question is yes, due to the seminal work of Choquet-Bruhat [34] (a presentation in book form is also available in, e.g., [82]):

Theorem 1 Given initial data $(\Sigma, \rho, \kappa)$ to Einstein's vacuum equations, there is a globally-hyperbolic development.

Clearly, this is a fundamental result. In particular, this result is what justifies the terminology "initial data to Einstein's vacuum equations" as specified in Definition 1. On the other hand, the issue of uniqueness is not addressed. Given initial data, there are infinitely many distinct globallyhyperbolic developments. In order to obtain uniqueness, it is consequently necessary to require some form of maximality.

\subsubsection{Existence of a maximal globally-hyperbolic development}

The central concept in the study of uniqueness is that of an MGHD:

Definition 3 Given initial data to Einstein's vacuum equations (5), a MGHD of the data is a globally hyperbolic development $(M, g)$, with embedding $i: \Sigma \rightarrow M$, such that if $\left(M^{\prime}, g^{\prime}\right)$ is any other globally hyperbolic development of the same data, with embedding $i^{\prime}: \Sigma \rightarrow M^{\prime}$, then there is a map $\psi: M^{\prime} \rightarrow M$, which is a diffeomorphism onto its image, such that $\psi^{*} g=g^{\prime}$ and $\psi \circ i^{\prime}=i$.

Note that this definition differs from the standard notion of maximality used in set theory. The standard notion would lead to the definition of a MGHD as a globally hyperbolic development, which cannot be extended (note that this notion of maximality would not a priori rule out the possibility of two maximal elements, neither of which can be embedded into the other, as opposed to Definition 3).

Theorem 2 Given initial data to Equation (5), there is an MGHD of the data, which is unique up to isometry.

Remark. Uniqueness of a development $(M, g)$ up to isometry is defined as follows: if $\left(M^{\prime}, g^{\prime}\right)$ is another MGHD, then there is a diffeomorphism $\psi: M \rightarrow M^{\prime}$ such that $\psi^{*} g^{\prime}=g$ and $\psi \circ i=i^{\prime}$, where $i$ and $i^{\prime}$ are the embeddings of $\Sigma$ into $M$ and $M^{\prime}$ respectively.

Theorem 2 is due to the work of Choquet-Bruhat and Geroch; see [13] for the original paper and [82] for a recent presentation. The proof relies, in part, on the local theory and on an argument using what is often referred to as Zorn's lemma. This leads to the existence of an MGHD in the set theory sense of the word. However, it does not lead to the existence of an MGHD in the sense

Living Reviews in Relativity

http: //www . livingreviews . org/lrr-2010-2 
of Definition 3. In fact, the important part of the result is the uniqueness of the MGHD (in the set theory sense of the word). This requires an additional argument.

Due to Theorem 2, the initial value formulation of Einstein's equations is meaningful. However, the MGHD might be extendible. In fact, it turns out that there are initial data such that this is the case. If the extensions were unique in their turn, this would not be a serious problem, but it turns out that there are MGHDs with inequivalent maximal extensions [20] (see also [82]). The reason these examples are unfortunate is that they demonstrate that Einstein's general theory of relativity is not deterministic; given initial data, there is not necessarily a unique corresponding universe. Nevertheless, the examples of this pathological behavior are very special, and there is thus reason to hope that for generic initial data, the MGHD is inextendible. These speculations naturally lead us to the strong cosmic-censorship conjecture.

\subsection{Strong cosmic censorship}

By the strong cosmic-censorship conjecture, we mean the following statement:

Conjecture 1 For generic asymptotically-flat and for generic spatially-compact initial data for Einstein's vacuum equations, the MGHD is inextendible.

Remark. We are only interested here in initial data specified on compact manifolds, a case to which the conjecture applies. Readers interested in the asymptotically-flat case are referred to, e.g., [90] and references cited therein.

In this form, the statement is due to Chruściel; see [17, Section 1.3], based on ideas due to Eardley and Moncrief [32]. It is of course also possible to make the same (or similar) statements in the presence of matter. However, there are some matter models, which exhibit pathologies, and we do not wish to discuss such issues here. The formulation of Conjecture 1 is rather vague; the words "generic" and "inextendible" occur without having been clearly defined. The reason for this is partly that there is no a priori preferred definition of these concepts. Let us discuss them separately.

\subsubsection{Genericity}

In the context of a finite-dimensional dynamic system, a generic subset could, e.g., be defined in one of the following ways:

- a set whose complement has measure zero (with respect to the Lebesgue measure, say),

- a set that is open and dense,

- a dense $G_{\delta}$ set, i.e., a countable intersection of open sets, which is dense.

Other possibilities are conceivable; see, e.g., [14]. Regardless of the choice of definition, one requirement appears to be quite clear: if a set is generic, then the complement should not be generic. In the case of infinite-dimensional dynamic systems, the case we are interested in here, the measure of the theoretic notion of genericity is not so natural. Consequently, we shall here, unless otherwise stated, take generic to mean open and dense. Nevertheless, such a definition is still not precise; it requires the prior definition of a topology on the set of initial data.

\subsubsection{Inextendibility}

Turning to the meaning of inextendibility, there are several possibilities. First, the inextendibility should refer to a particular differentiability class; a solution could be extendible in one degree of differentiability but inextendible in another (that such a situation can occur is illustrated by [23, 24]). Furthermore, one could say that a solution is extendible if 
- it is extendible as a Lorentz manifold, or

- it is extendible as a Lorentz manifold solving Einstein's equations.

Here, we shall say that a solution is extendible if it is extendible as a Lorentz manifold. In other words, we shall not require the extension to be a solution to Einstein's equations. Furthermore, we shall use the differentiability class $C^{2}$, since we wish the differentiability class to be strong enough that curvature is still defined.

\subsection{Curvature blow up}

The strong cosmic-censorship conjecture is of fundamental importance since it expresses the expectation that Einstein's general theory of relativity is a deterministic theory (with some nongeneric exceptions). On the other hand, it is of a somewhat philosophical nature. However, it is strongly connected to a statement concerning the behavior of gravitational fields close to the singularity. Here, the existence of a singularity is equated with the existence of an incomplete causal geodesic, the motivation being the work of Hawking and Penrose resulting in the singularity theorems; see $[61,41,43]$ and $[42,89,60]$. Even though the commonplace existence of singularities is established by the singularity theorems, their nature remains unclear; do, for example, the gravitational fields become arbitrarily strong in the vicinity of a singularity? It is natural to state the following conjecture:

Conjecture 2 For generic asymptotically-flat and generic spatially-compact initial data for Einstein's vacuum equations, curvature blows up in the incomplete directions of causal geodesics in the $M G H D$.

In the statement of this conjecture, we shall in practice take curvature blow up to mean the unboundedness of the Kretschmann scalar,

$$
R_{\alpha \beta \gamma \delta} R^{\alpha \beta \gamma \delta}
$$

Of course, in the presence of matter one could also consider the contraction of the Ricci tensor with itself. Needless to say, there are many other possibilities. Again, the word "generic" has to be included. The reason is that the canonical counterexample to inextendibility of the MGHD in the cosmological vacuum case, Taub-NUT, is also a counterexample to curvature blow up in the incomplete directions of causal geodesics. Taub-NUT is causally geodesically-incomplete both to the future and to the past, but the spacetime can be extended and all curvature invariants consequently remain bounded along all geodesics; see [20] or [82]. The reason for wanting to prove Conjecture 2 is perhaps more clear than the reason for wanting to prove strong cosmic censorship; it would demonstrate the generic occurrence of singularities, not only in the sense of causal geodesic completeness, but in the sense of the gravitational fields becoming arbitrarily strong. Furthermore, if Conjecture 2 holds, then strong cosmic censorship follows (with the $C^{2}$ concept of inextendibility).

Since it is not clear how to address Conjectures 1 and 2 in all generality, the work that has been carried out so far has been concerned with the analogous questions phrased in the context of special classes of spacetimes. Here, we shall be concerned with these questions phrased in the Gowdy class.

\subsection{Pathological examples in the case of Gowdy}

Before ending this section, let us record the existence of pathological behavior in the class of Gowdy spacetimes. The most obvious example is provided by the flat Kasner solution; letting

Living Reviews in Relativity

http: //www . livingreviews . org/lrr-2010-2 
$\lambda(t, \theta)=P(t, \theta)=\ln t$ in Equation (4) leads to the metric

$$
g_{F K}=-d t^{2}+d \theta^{2}+t^{2} d \sigma^{2}+d \delta^{2} .
$$

Viewing this metric on $(0, \infty) \times \mathbb{R}^{3}$,

$$
\phi_{K}(t, \theta, \sigma, \delta)=(t \cosh \sigma, \theta, t \sinh \sigma, \delta)
$$

yields an isometry to a subset of Minkowski space. Consequently, $g_{F K}$ is flat, and we shall refer to $\left[(0, \infty) \times \mathbb{R}^{3}, g_{F K}\right]$ as the flat Kasner solution. This solution is past causally geodesically incomplete, but the curvature is clearly bounded. Furthermore, it is extendible. Considering the same solution on the torus, it is also possible to construct an extension; see, e.g., [82].

However, there are more sophisticated examples of pathologies. In [20], the authors demonstrate that given any positive integer $n$, there is a polarized Gowdy vacuum spacetime with at least $n$ inequivalent maximal extensions. 


\section{BKL, Fuchsian Methods and Asymptotic Expansions}

The results on strong cosmic censorship in Gowdy vacuum spacetimes cover the polarized sub-case as well as the general $T^{3}$-Gowdy case. However, to the best of our knowledge, there are no results concerning strong cosmic censorship in the general $S^{3}$ and $S^{2} \times S^{1}$ cases. The method of proof, in all the situations in which results exist, consists of a detailed analysis of the asymptotics of solutions. As a consequence, we shall devote most of this review to a description of the analysis of the asymptotic behavior. Note that it might be possible to prove strong cosmic censorship without analyzing the asymptotics in detail. In fact, there are proofs under related symmetry assumptions, which are not based on a detailed analysis of the asymptotics [25, 26, 27, 84].

The existence of asymptotic expansions in the direction towards the singularity has played a central role in proving strong cosmic censorship for $T^{3}$ and polarized vacuum Gowdy spacetimes. In the latter case, to take one example, there is a computation of asymptotic expansions due to Isenberg and Moncrief [50]. This computation was then used in [21] in the proof of strong cosmic censorship in the polarized case. In the general $T^{3}$-case, there is a large literature on asymptotic expansions, which we shall return to in Section 8; the starting point being the work of Grubišić and Moncrief [40]. It is worth noting that both in the case of [40] and [50], the ideas of Belinskii, Khalatnikov, and Lifshitz [55, 6, 7] (henceforth BKL) played an important role. As a consequence, we wish to give a brief description of the BKL perspective as well as of some related proposals.

\subsection{The BKL picture}

The ideas of BKL are often referred to as "the BKL conjecture". This terminology is unfortunate, since it would seem to indicate that there is a mathematically precise statement that can be proven or disproven. In reality, the ideas are too vague to warrant the name "conjecture". Nevertheless, they have played an important role on an intuitive level, and therefore deserve to be mentioned. The rough idea is as follows:

- given a spacetime $(M, g)$ such that all past-directed timelike geodesics are incomplete, there is a spacelike hypersurface $\Sigma$ such that the past of $\Sigma$ is diffeomorphic to $\left(0, t_{0}\right] \times \Sigma$ for some $t_{0}>0$, where the first coordinate in the division $\left(0, t_{0}\right] \times \Sigma$ defines a time function,

- as $t \rightarrow 0+$, different spatial points do not causally influence each other, i.e., if $p, q \in \Sigma$ and $p \neq q$, then, for $t$ small enough, the past of $(t, p)$ does not intersect the past of $(t, q)$,

- the matter content is of negligible importance for the dynamics as $t \rightarrow 0+$,

- "time derivatives" (or "kinetic terms") dominate "spatial derivatives" (or "spatial curvature coupling terms") as $t \rightarrow 0+$,

- for a fixed $p \in \Sigma$, the behavior of the solution along $(t, p)$ is well approximated by a spatiallyhomogeneous vacuum solution, in particular by an oscillatory solution (Bianchi types VIII, IX and $\left.\mathrm{VI}_{-1 / 9}\right)$.

There are some caveats. First, the statements concern generic spacetimes; there are exceptions; see Taub-NUT. Second, the matter content might be important for special classes of matter models. For instance, in the case of a stiff fluid or a scalar field, the matter should play a dominant role. The statement that solutions should exhibit oscillatory behavior also depends on the matter model; stiff fluids and scalar fields are expected to suppress it. Furthermore, symmetry might prevent the appearance of oscillations.

The first statement on the list above can be ensured under general circumstances; a combination of Hawking's theorem [60, Theorem 55A, p. 431], an energy condition and the existence of a Cauchy

Living Reviews in Relativity

http: //www. livingreviews.org/lrr-2010-2 
hypersurface satisfying suitable assumptions concerning the mean curvature will do. The statement of causal disconnectedness is, however, unsatisfactory in that it depends not only on the foliation, but also on a choice of diffeomorphism. It would be preferable to have a geometric condition, which is even independent of the foliation. However, to our knowledge there is no such definition; see the introduction of [44] for a further discussion. The last three statements are clearly very vague.

The general framework has been developed significantly since the work of BKL; see [28, 30, $47,88]$ and references cited therein. We shall not describe these developments in detail, but let us mention that there are at least two somewhat different approaches. In the Hamiltonian approach, taken in [28,30], billiards describe the asymptotic behavior (see also the work of Misner and Chitré; see [57, pp. 805-816] and references cited therein). In the dynamic systems approach, described in [47,88], the solution is approximated asymptotically by a family of solutions to ordinary differential equations. In [3], the Gowdy spacetimes have been considered from this perspective.

Even though the two perspectives have differences, they have an essential assumption in common: causal disconnectedness in the direction toward the singularity. Furthermore, in both cases, the oscillatory spatially-homogeneous vacuum solution is of central importance. It is of interest to note that these two aspects are potentially contradictory; Misner's original motivation in studying the Mixmaster Universe (Bianchi IX) [56] was the desire to demonstrate that there is no causal disconnectedness in the direction towards the singularity. In order for the pictures suggested in $[28,30]$ and $[47,88]$ to be consistent, causal disconnectedness should hold in the oscillatory spatially-homogeneous vacuum solutions. It is far from clear that this is the case. The reader interested in a discussion of the status of this question is referred to the introduction of [44].

Finally, let us mention that a different formulation of the BKL picture is given in [19, Conjecture 6.10, p. 58]; see also the references cited therein.

\subsection{Asymptotic expansions, Fuchsian methods}

It is interesting to note that, in spite of the fact that the BKL picture and related proposals emphasize the importance of oscillatory behavior, the greatest successes of the BKL point of view have been obtained in the nonoscillatory setting. The main reason is that in the absence of oscillations, it is sometimes possible to characterize the asymptotic behavior in terms of asymptotic data (of course, the lack of results in the presence of oscillations is largely due to the difficulty in analyzing the asymptotic behavior in that case). In certain situations, this characterization is strong enough that the asymptotic data are in one-to-one correspondence with the solutions. If that is the case, the asymptotic data can be considered to be "initial data at the singularity". The results in the nonoscillatory case come in different forms.

\subsubsection{From solutions to asymptotics}

The type of result, that is of greatest immediate interest is the one that, given a solution to the Einstein equations, provides asymptotic expansions. The means by which this is achieved vary. One method is to devise a simplified system of equations, such that the solution to the Einstein equations converges to a solution to the simplified system. In the spirit of the BKL picture, the simplified system is often obtained by omitting some (if not all) spatial derivatives. One example of a successful application is given by the analysis of Isenberg and Moncrief [50] in the polarized Gowdy case (which in some respects follows the ideas of [31]). In [50], a simplified system consisting of the Velocity Term Dominated (VTD) equations are introduced and solved, and the authors prove that solutions to the Einstein equations converge to solutions to the VTD system. Furthermore, a geometric definition for what the authors call Asymptotically Velocity-Term Dominated near the Singularity (or AVTDS) is given; see [50, pp. 88-89]. We give a brief description of the analysis of [50] in Section 7. 


\subsubsection{From asymptotics to solutions}

Another type of result starts by specifying the asymptotics at the singularity and then proceeds to prove that there are solutions with these asymptotics. The analysis is based on reducing the particular form of the Einstein equations under consideration to an equation in Fuchsian form:

$$
t \partial_{t} u+A(x) u=f\left(t, x, u, u_{x}\right)
$$

see [29, (1.5), p. 1054]; see also [53]. There is a standard theory, which deals with equations of the form of Equation (10), even with the nonregular problem of specifying initial data at $t=0$, which is the case of interest in the present context. Consequently, the central problem is that of reformulating Einstein's equations to Fuchsian form. The general procedure is as follows:

- express Einstein's equations with respect to a suitable gauge (in the case of $T^{3}$-Gowdy for instance, the areal time coordinate has turned out to be a good candidate; see [54, 68], and in the cases without symmetries concerning which results have been obtained, a Gaussian time coordinate has proven useful [2, 29]),

- identify the leading-order asymptotic behavior, where the leading-order terms preferably should correspond to as many free functions as are required to specify regular initial data (in the case of $T^{3}$-Gowdy, formal expansions had been suggested in [40] prior to [54] and in $[2,29]$, the expansions were obtained by considering "Velocity Term Dominated" systems associated with the full system of Einstein's equations),

- express the unknowns in terms of the leading-order terms plus a remainder, and write down the equations in terms of the remainder (this equation should be of Fuchsian form),

- apply the Fuchsian theory.

The standard Fuchsian theory is applicable in the real analytic setting. As a consequence, most of the results assume real analytic "data at the singularity" and lead to the conclusion that there are real analytic solutions with the corresponding asymptotic behavior. Clearly, the procedure is not always applicable. In particular, it is not expected to be applicable in the presence of oscillations.

\subsubsection{Overview of results}

Let us mention some of the results that have been obtained using Fuchsian methods. In [54], Fuchsian methods were applied to the $T^{3}$-Gowdy case in the real analytic setting. The assumption of real analyticity was later relaxed to smoothness [68]. See also [86] for a similar analysis in the $S^{2} \times S^{1}$ and $S^{3}$ cases (though there are some problems related to the symmetry axes in that case, and as a consequence, the results are less complete). An analysis of the polarized $T^{2}$-symmetric spacetimes in the real analytic setting was carried out in [48]. In all the examples mentioned so far, the symmetry caused the suppression of oscillations. However, matter can also have the same effect. This is illustrated by [2], which consists of a study of the Einstein equations coupled to either a scalar field or a stiff fluid. The results are in the real analytic setting and associate a solution to asymptotic initial data. Finally, in [29] large classes of matter models are considered in various dimensions with similar results. 


\section{Equations}

In our presentation, we shall focus on $T^{3}$-Gowdy. Therefore, we will only write down here the equations in that case. The reader interested in the equations for polarized $S^{2} \times S^{1}$ or $S^{3}$-Gowdy is referred to [50, (12a) - (12c), p. 92].

\subsection{Expanding direction}

The starting point is the metric (2). For metrics of this form, Einstein's vacuum equations are equivalent to

$$
\begin{aligned}
P_{t t}+\frac{1}{t} P_{t}-P_{\theta \theta}-e^{2 P}\left(Q_{t}^{2}-Q_{\theta}^{2}\right) & =0, \\
Q_{t t}+\frac{1}{t} Q_{t}-Q_{\theta \theta}+2\left(P_{t} Q_{t}-P_{\theta} Q_{\theta}\right) & =0,
\end{aligned}
$$

and

$$
\begin{aligned}
& \lambda_{t}=t\left[P_{t}^{2}+P_{\theta}^{2}+e^{2 P}\left(Q_{t}^{2}+Q_{\theta}^{2}\right)\right], \\
& \lambda_{\theta}=2 t\left(P_{\theta} P_{t}+e^{2 P} Q_{\theta} Q_{t}\right) .
\end{aligned}
$$

Since the equations for $P$ and $Q$ do not depend on $\lambda$, it is possible to solve Equations (11) - (12) first and then to calculate $\lambda$ using Equation (14). In order for this procedure to be meaningful, it is necessary to choose the initial data for $P$ and $Q$ in such a way that the integral of the right-hand side of Equation (14) vanishes. However, assuming that has been done, $\lambda$ is determined up to a constant given a solution to Equations (11)-(12).

\subsection{The direction towards the singularity}

In the study of the direction towards the singularity, it is convenient to change the time coordinate to $t=e^{-\tau}$, so that the singularity corresponds to $\tau \rightarrow \infty$. The metric then becomes

$$
g_{T^{3}}=e^{(\tau-\lambda) / 2}\left(-e^{-2 \tau} d \tau^{2}+d \theta^{2}\right)+e^{-\tau}\left[e^{P} d \sigma^{2}+2 e^{P} Q d \sigma d \delta+\left(e^{P} Q^{2}+e^{-P}\right) d \delta^{2}\right] .
$$

Here, $\tau \in \mathbb{R}$ and $(\theta, \sigma, \delta)$ are coordinates on $T^{3}$. Einstein's vacuum equations take the form

$$
\begin{aligned}
P_{\tau \tau}-e^{-2 \tau} P_{\theta \theta}-e^{2 P}\left(Q_{\tau}^{2}-e^{-2 \tau} Q_{\theta}^{2}\right) & =0, \\
Q_{\tau \tau}-e^{-2 \tau} Q_{\theta \theta}+2\left(P_{\tau} Q_{\tau}-e^{-2 \tau} P_{\theta} Q_{\theta}\right) & =0,
\end{aligned}
$$

and

$$
\begin{aligned}
& \lambda_{\tau}=P_{\tau}^{2}+e^{-2 \tau} P_{\theta}^{2}+e^{2 P}\left(Q_{\tau}^{2}+e^{-2 \tau} Q_{\theta}^{2}\right), \\
& \lambda_{\theta}=2\left(P_{\theta} P_{\tau}+e^{2 P} Q_{\theta} Q_{\tau}\right) .
\end{aligned}
$$

\subsection{Wave-map structure}

It is of interest to note that Equations (16)-(17) are of wave-map type. The domain is given by $\mathbb{R} \times T^{2}$ with the metric

$$
g_{0}=-e^{-2 \tau} d \tau^{2}+d \theta^{2}+e^{-2 \tau} d \chi^{2}
$$

and the target is $\mathbb{R}^{2}$ with the metric

$$
g_{R}=d P^{2}+e^{2 P} d Q^{2} .
$$


Equations (16)- (17) are the wave-map equations for a map from $\left(\mathbb{R} \times T^{2}, g_{0}\right)$ to $\left(\mathbb{R}^{2}, g_{R}\right)$, which is independent of the $\chi$-coordinate. In other words, they coincide with the Euler-Lagrange equations corresponding to the action

$$
\iint\left[-P_{\tau}^{2}-e^{2 P} Q_{\tau}^{2}+e^{-2 \tau}\left(P_{\theta}^{2}+e^{2 P} Q_{\theta}^{2}\right)\right] d \theta d \tau
$$

\subsubsection{Representations of hyperbolic space}

It is of interest to note that $\left(\mathbb{R}^{2}, g_{R}\right)$ is isometric to hyperbolic space. In fact,

$$
\phi_{R H}(Q, P)=\left(Q, e^{-P}\right)
$$

gives an isometry from $\left(\mathbb{R}^{2}, g_{R}\right)$ to the upper half plane model of hyperbolic space, $\left(H, g_{H}\right)$, where $H=\left\{(x, y) \in \mathbb{R}^{2}: y>0\right\}$ and

$$
g_{H}=\frac{d x^{2}+d y^{2}}{y^{2}} .
$$

For certain considerations, the upper half plane is an inappropriate model of hyperbolic space. The reason is that the upper half plane has a preferred boundary point, namely infinity; see Section 9.3. The disc model, given by the Riemannian metric

$$
g_{D}=\frac{4\left(d x^{2}+d y^{2}\right)}{\left(1-x^{2}-y^{2}\right)^{2}},
$$

on the open unit disc $D$ does not have preferred boundary points, and therefore is sometimes preferable. Using complex notation,

$$
\phi_{H D}=\frac{z-i}{z+i}
$$

yields an isometry from the upper half plane to the disc model. By composing $\phi_{H D}$ with $\phi_{R H}$, we obtain an isometry from $\left(\mathbb{R}^{2}, g_{R}\right)$ to $\left(D, g_{D}\right)$. The expression is given by

$$
\phi_{R D}(Q, P)=\frac{Q+i\left(e^{-P}-1\right)}{Q+i\left(e^{-P}+1\right)} .
$$

\subsection{Conserved quantities, kinetic energy density}

The fact that Equations (11) - (12) are wave-map equations has some important consequences. In particular, since the target has a three-dimensional isometry group, there should be three conserved quantities. In fact, there are constants $A, B$ and $C$ such that

$$
\begin{aligned}
A & =\int_{S^{1}}\left\{2 Q\left(t Q_{t}\right) e^{2 P}-2\left(t P_{t}\right)\right\} d \theta \\
B & =\int_{S^{1}} e^{2 P}\left(t Q_{t}\right) d \theta \\
C & =\int_{S^{1}}\left\{\left(t Q_{t}\right)\left(1-e^{2 P} Q^{2}\right)+2 Q\left(t P_{t}\right)\right\} d \theta .
\end{aligned}
$$

That the derivatives of the right-hand sides of these expressions are zero can be verified using Equations (11)-(12).

Another important consequence of the wave-map structure is the fact that isometries of hyperbolic space map solutions to solutions. 
Finally, it is convenient to introduce the kinetic energy density,

$$
\mathcal{K}=P_{\tau}^{2}+e^{2 P} Q_{\tau}^{2}
$$

Note that this quantity is obtained by computing the time derivative of the wave map and then taking the squared length of the resulting vector field using the target metric. As a consequence, the kinetic energy is a geometric object. In other words, two solutions related by an isometry have the same kinetic energy density. 


\section{Singularity, Polarized Case}

The proof of strong cosmic censorship, in the polarized as well as in the $T^{3}$-Gowdy case, proceeds via Conjecture 2. In other words, it consists of a proof of the fact that, generically, the curvature is unbounded in the incomplete directions of causal geodesics. In the polarized case with $S^{3}$ and $S^{2} \times S^{1}$ topology, the causal geodesics can be proven to be incomplete both to the future and to the past [21, p. 1673]. Thus, in those cases it is only necessary to analyze the singularities. In the case of $T^{3}$-Gowdy, there is an expanding direction, and it is necessary to prove that causal geodesics are complete in that direction. In general, it is thus necessary to analyze the behavior in the direction towards the singularity and the behavior in the expanding direction. Since the methods involved are very different, we shall consider the two cases separately. Furthermore, since the analysis in the polarized and general cases are quite different, we shall begin by describing the analysis in the direction towards the singularity in polarized Gowdy.

\subsection{Equations, polarized $T^{3}$-Gowdy}

In the case of polarized Gowdy, $Q=0$, so that Equations (16)-(19) reduce to

$$
\begin{aligned}
P_{\tau \tau}-e^{-2 \tau} P_{\theta \theta} & =0 \\
\lambda_{\tau} & =P_{\tau}^{2}+e^{-2 \tau} P_{\theta}^{2}, \\
\lambda_{\theta} & =2 P_{\theta} P_{\tau} .
\end{aligned}
$$

As an aside, it is of interest to note that the set $Q=0$ coincides with the image of a geodesic in hyperbolic space $\left(\mathbb{R}^{2}, g_{R}\right)$. This system of equations should be compared with (11a)-(11d) of [50] (note, however, that the variables of [50] are somewhat different).

\subsection{Associated Velocity Term Dominated system}

Proceeding along the lines of [50], let us define the associated VTD system given by

$$
\begin{aligned}
P_{\mathrm{VD}, \tau \tau} & =0 \\
\lambda_{\mathrm{VD}, \tau} & =P_{\mathrm{VD}, \tau}^{2}, \\
\lambda_{\mathrm{VD}, \theta} & =2 P_{\mathrm{VD}, \theta} P_{\mathrm{VD}, \tau} ;
\end{aligned}
$$

see [50, (13a)-(13d), p. 92]. Clearly, this system can be integrated explicitly. In particular,

$$
P_{\mathrm{VD}}(\tau, \theta)=v_{\mathrm{VD}}(\theta) \tau+\phi_{\mathrm{VD}}(\theta)
$$

for some smooth functions $v_{\mathrm{VD}}(\theta)$ and $\phi_{\mathrm{VD}}$. Given this information, $\lambda_{\mathrm{VD}}$ can then be calculated.

\subsection{Asymptotics of the solution to the polarized $T^{3}$-Gowdy equations}

Let us briefly illustrate how one can obtain conclusions concerning solutions to Equations (29) - (31) similar to those obtained for the VTD system. Define the energies as

$$
E_{k}=\frac{1}{2} \sum_{j \leq k-1} \int_{S^{1}}\left[\left(\partial_{\theta}^{j} \partial_{\tau} P\right)^{2}+e^{-2 \tau}\left(\partial_{\theta}^{j+1} P\right)^{2}\right] d \theta
$$

for $k \geq 1$. Differentiating with respect to $\tau$, integrating by parts and using Equation (29) leads to the conclusion that the energies $E_{k}$ are decaying. Combining this observation with Sobolev embedding leads to the conclusion that, regardless of the choice of $j, \partial_{\theta}^{j} \partial_{\tau} P$ is bounded to the

Living Reviews in Relativity

http://www. livingreviews . org//rr-2010-2 
future. Consequently, regardless of the choice of $j, \partial_{\theta}^{j} P$ does not grow faster than linearly to the future. Inserting this information into Equation (29) and integrating yields two smooth functions $v$ and $\phi$ such that

$$
P(\tau, \theta)=v(\theta) \tau+\phi(\theta)+u(\tau, \theta), \quad P_{\tau}(\tau, \theta)=v(\theta)+w(\tau, \theta),
$$

where $u$ and $w$ are functions such that they and all their spatial derivatives are $O\left(\tau e^{-2 \tau}\right)$. In other words, the leading-order behavior is given by the solution to the VTD equations, and the VTD equations, in their turn, are obtained by essentially dropping the spatial derivatives. The above analysis should be compared with [50, Theorem III.5, pp. 102-103].

\subsection{Curvature blow up, polarized $T^{3}$-case}

From the point of view of proving strong cosmic censorship, the main reason for wanting to derive expansions of the form of Equation (35) is that they can be used to prove curvature blow up. It turns out that criteria for curvature blow up and the absence thereof can be formulated in terms of $v$. Consider a past inextendible causal curve $\gamma$. Due to the form of the metric (2), it is clear that the $\theta$-coordinate of this curve has to converge in the direction towards the singularity. Call the limiting value $\theta_{0}$. Then, if

- $v^{2}\left(\theta_{0}\right) \neq 1$,

- $v^{2}\left(\theta_{0}\right)=1$ but $\partial_{\theta} v\left(\theta_{0}\right) \neq 0$, or

- $v^{2}\left(\theta_{0}\right)=1, \partial_{\theta} v\left(\theta_{0}\right)=0$, but $\partial_{\theta}^{2} v\left(\theta_{0}\right) \neq 0$,

the Kretschmann scalar is unbounded along $\gamma$ in the direction of the singularity. Otherwise, it is bounded. This result, as well as quantitative estimates for the rate at which the curvature tends to infinity, is contained in [50, Theorem IV.1, p. 105].

\subsection{Asymptotic velocity, polarized $T^{3}$-case}

It is of interest to note that the quantity $v$, or, more precisely, its absolute value, has a geometric significance. Viewing Equations (16)-(17) as solutions to the wave-map equation, the kinetic energy density $\mathcal{K}$ is a geometric object; see Section 6.4. Furthermore, due to the asymptotics of Equation (35), we have

$$
v^{2}(\theta)=\lim _{\tau \rightarrow \infty} \mathcal{K}(\tau, \theta) .
$$

Consequently, $v^{2}$ is a geometric object. The quantity $|v|$ can also be characterized as the rate at which solutions tend to the boundary of hyperbolic space; if $x_{0}$ is a fixed point in hyperbolic space and the solution is represented by $x$, then

$$
|v(\theta)|=\lim _{\tau \rightarrow \infty} \frac{d_{H}\left(x(\tau, \theta), x_{0}\right)}{\tau},
$$

where $d_{H}$ is the topological metric induced on hyperbolic space by the hyperbolic metric. To conclude, $|v(\theta)|$ can be characterized geometrically when viewing Equations (16) - (17) as wavemap equations. Furthermore, in the polarized setting, its properties can be used to characterize curvature blow up. We shall loosely refer to $v$ as the velocity.

\section{6 $\quad S^{2} \times S^{1}$ and $S^{3}$ cases}

The $S^{3}$ and $S^{2} \times S^{1}$ cases are also treated in [50]. The results and the analysis are similar but more technical due to the presence of the axes. Consequently, we refer the interested reader to [50] for the details. 


\section{Asymptotic Expansions Using Fuchsian Methods, Gen- eral $T^{3}$-Case}

The analysis in the polarized case, which we outlined above, illustrates the importance of being able to compute asymptotic expansions. Thus, in analyzing the general case it is natural to begin by trying to carry out a similar computation. As we observed in Section 5.2, there are two different ways to proceed. One is to derive expansions given the solution. The other is to prove the existence of solutions with specified asymptotics. In the present section, we are concerned with the latter perspective.

The natural starting point for the exposition is the formal expansions in the general $T^{3}$-Gowdy case, which were derived in [40]. In [54], Fuchsian methods were then used to prove their existence. With respect to our parametrization of the metric, the expansions take the form

$$
\begin{aligned}
& P(\tau, \theta)=v_{a}(\theta) \tau+\phi(\theta)+u(\tau, \theta) \\
& Q(\tau, \theta)=q(\theta)+e^{-2 v_{a}(\theta) \tau}[\psi(\theta)+w(\tau, \theta)],
\end{aligned}
$$

where $w, u \rightarrow 0$ as $\tau \rightarrow \infty$ and $0<v_{a}(\theta)<1$. Let us comment on a few aspects of these expansions.

\subsection{Geometric interpretation of $v_{a}$}

Assuming that the derivatives of $u$ and $w$ with respect to time and space tend to zero, the expansions (37) - (38) imply (recall that $v_{a}>0$ )

$$
v_{a}(\theta)=\lim _{\tau \rightarrow \infty} \mathcal{K}^{1 / 2}(\tau, \theta),
$$

where the kinetic energy density $\mathcal{K}$ is given by Equation (28). In other words, from a geometric point of view, $v_{a}$ is the same object as in the polarized case. Furthermore, it is possible to prove that if expansions of the form of Equations (37)-(38) hold with $0<v_{a}\left(\theta_{0}\right)<1$, then the Kretschmann scalar is unbounded along causal curves whose $\theta$-component converges to $\theta_{0}$ in the direction towards the singularity; see Section 7.4. As in the polarized case, we shall refer to $v_{a}$ as the velocity. The condition $0<v_{a}<1$ will in the future be referred to as low velocity, whereas $v_{a} \geq 1$ will be referred to as high velocity.

\subsection{Restriction on the velocity}

Note that Equation (37) is, disregarding the difference in notation, identical to the first equation in (35). However, in the present setting, there is a restriction on $v_{a}$, which did not appear in the polarized case. The essential part of this restriction is the inequality $0<\left|v_{a}(\theta)\right|<1$; the sign of $v_{a}$ is not of central importance. The restriction $v_{a}<1$ is due to the fact that there is a potential inconsistency with Equation (16) if $v_{a} \geq 1$; assuming the derivatives of $u$ and $w$ with respect to time and space to tend to zero, all the terms in Equation (16) tend to zero except, possibly, $e^{2 P-2 \tau} Q_{\theta}^{2}$, which is roughly $e^{2\left(v_{a}(\theta)-1\right) \tau} q_{\theta}^{2}(\theta)$. In fact, if $v_{a}(\theta) \geq 1$ and $q_{\theta}(\theta) \neq 0$, the expansions are clearly inconsistent. Furthermore, considering Equation (16), the term $e^{2 P-2 \tau} Q_{\theta}^{2}$ would in that case seem to have the effect of causing $P_{\tau}$ to decrease. For a further discussion, see [10].

\subsection{Geodesic loop}

Consider a solution with asymptotics of the form of Equations $(37)-(38)$ and $v_{a}(\theta)>0$. Then $Q(\tau, \theta)$ converges and $P(\tau, \theta)$ tends to infinity as $\tau \rightarrow \infty$. In other words, for a fixed $\theta$, the solution roughly speaking goes to the boundary along a geodesic in hyperbolic space; see Equation (21). Since $P$ and $Q$, for a fixed $\tau$, define a loop in hyperbolic space, the solution is asymptotically approximated by a "loop of geodesics".

Living Reviews in Relativity

http: //www. livingreviews.org/lrr-2010-2 


\subsection{Existence of expansions using Fuchsian methods, $T^{3}$-case}

In [54], Kichenassamy and Rendall proved the existence of expansions in the real analytic setting. In other words, they proved that, given real analytic $v_{a}, \phi, q, \psi$ with $0<v_{a}<1$, there is a unique solution to Equations (16) - (17) with expansions of the form of Equations (37)-(38). Note that the number of functions that are freely specifiable in the expansions coincides with the number of functions that need to be specified in order to obtain a unique solution to the initial value problem corresponding to Equations (16)-(17). For reasons mentioned in Section 8.2, the expansions suffer from a potential consistency problem in the case of $v_{a} \geq 1$ and $q_{\theta} \neq 0$. However, in [54] it was proven that if $q$ is constant, the condition on $v_{a}$ can be relaxed to $v_{a}>0$. The regularity condition of [54] was relaxed to smoothness in [68].

\subsection{Existence of expansions using Fuchsian methods, $S^{2} \times S^{1}$ and $S^{3}$ cases}

There are results concerning the existence of expansions in the $S^{2} \times S^{1}$ and $S^{3}$ cases as well [86]. However, the analysis is complicated by the presence of the axes; regularity conditions lead to the requirement that the velocity has to be either -1 or 3 there. As a consequence, the result is different, since such values of the velocity are inconsistent with expansions containing the full number of free functions. We refer the reader interested in more details to [86]. 


\section{Spikes}

Numerical studies of solutions to Equations (16)-(17) indicate that for most spatial points, behavior similar to that described by the asymptotic expansions (37) - (38) occurs [11, 10]. However, the studies also indicate that there are exceptional spatial points at which the behavior is different. Due to the appearance of the solutions in the neighborhood of the exceptional points, the corresponding features have been referred to as "spiky features" or "spikes". Their existence would seem to necessitate an understanding of the "spikes" on an analytical level in order to be able to describe the asymptotics of general $T^{3}$-Gowdy solutions. An important step in this direction was achieved by demonstrating the existence of a large class of solutions to Equations (16)-(17) with spikes [71]. In order to be able to describe these solutions, we need to introduce some transformations taking solutions to solutions.

\subsection{Inversion}

As was explained in Section 6.3, Equations (16)-(17) can be viewed as wave-map equations with hyperbolic space as a target. As a consequence, isometries of hyperbolic space map solutions to solutions. In the context of spikes, one isometry of $\left(\mathbb{R}^{2}, g_{R}\right)$, which is particularly important (as was noted in [71]) is

$$
\operatorname{Inv}\left(Q_{0}, P_{0}\right)=\left[\frac{Q_{0}}{Q_{0}^{2}+e^{-2 P_{0}}}, P_{0}+\ln \left(Q_{0}^{2}+e^{-2 P_{0}}\right)\right] .
$$

We shall refer to this isometry as an inversion; in the upper half plane, it corresponds to an inversion in the unit circle with center at the origin.

\subsection{Gowdy to Ernst transformation}

In the construction of spikes, the Gowdy to Ernst transformation [71, p. 2963] will play an important role. Let $(Q, P)$ be a solution to Equations $(16)-(17)$ with $\theta \in \mathbb{R}$. In other words, the solution need not be periodic in the spatial variable. Then, up to a constant translation in $Q_{1}$, two smooth functions $Q_{1}$ and $P_{1}$ are defined by

$$
P_{1}=\tau-P, \quad Q_{1 \tau}=-e^{2(P-\tau)} Q_{\theta}, \quad Q_{1 \theta}=-e^{2 P} Q_{\tau} .
$$

Note that Equation (17) ensures that the definitions of $Q_{1 \tau}$ and $Q_{1 \theta}$ are compatible. Furthermore, $\left(Q_{1}, P_{1}\right)$ solve Equations (16) - (17). Assuming $Q_{1}\left(\tau_{0}, \theta_{0}\right)=q_{0}$, we shall denote the corresponding transformation, defined by Equation (41), by $\mathrm{GE}_{q_{0}, \tau_{0}, \theta_{0}}$ :

$$
\left(Q_{1}, P_{1}\right)=\mathrm{GE}_{q_{0}, \tau_{0}, \theta_{0}}(Q, P) .
$$

We shall refer to this transformation as the Gowdy to Ernst transformation. Note that, even if $(Q, P)$ is periodic in $\theta$, the same need not be true of $\left(Q_{1}, P_{1}\right)$. However, here we are mainly interested in local (in space) properties of the solutions, and, therefore, this aspect is not important.

\subsection{False spikes}

One way of constructing a spike is to start with a solution $(Q, P)$ with asymptotic expansions of the form of Equations (37) - (38), where $0<v_{a}<1$ (due to [68], we know that such solutions exist and that we are free to specify the functions $v_{a}, \phi, q, \psi$, assuming $0<v_{a}<1$ ). Assume that $q$ has the properties that $q\left(\theta_{0}\right)=0$ and that $q \neq 0$ in the punctured neighborhood of $\theta_{0}$. Applying an inversion to $(Q, P)$, we obtain

$$
\left(Q_{1}, P_{1}\right)=\operatorname{Inv}(Q, P)
$$




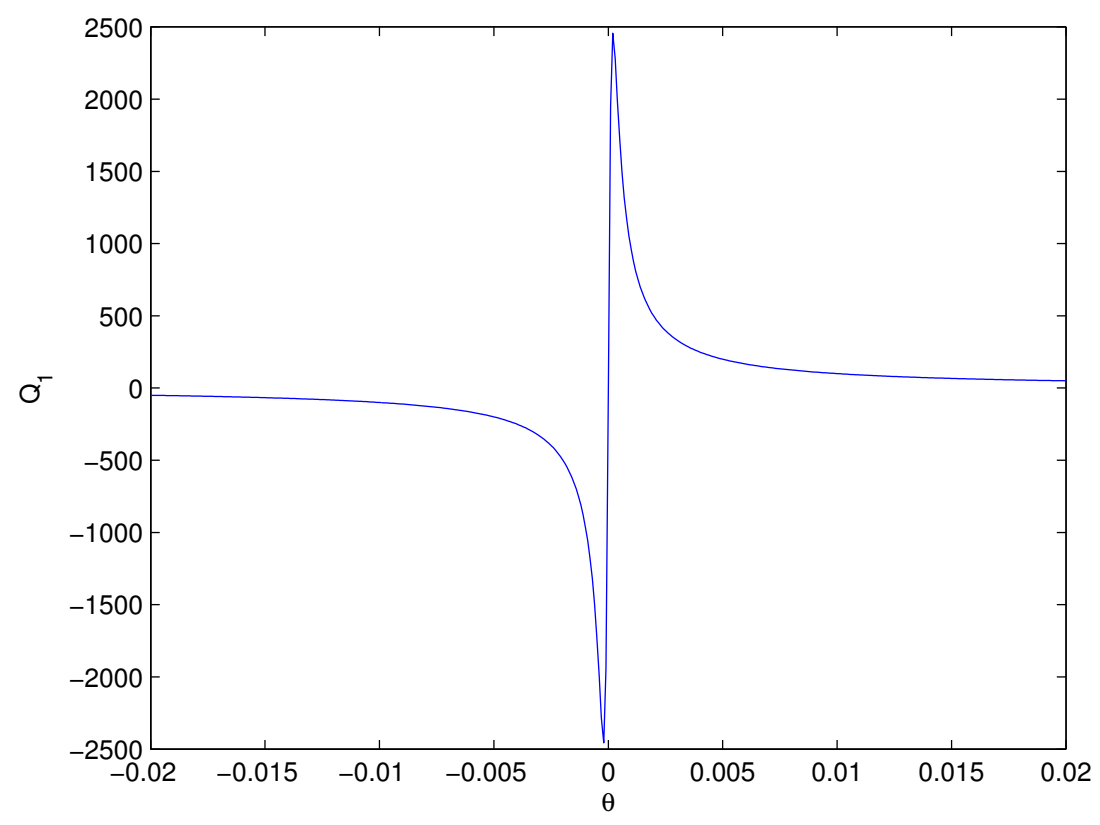

Figure 1: $Q_{1}$ in the neighborhood of a false spike for a fixed $\tau$.

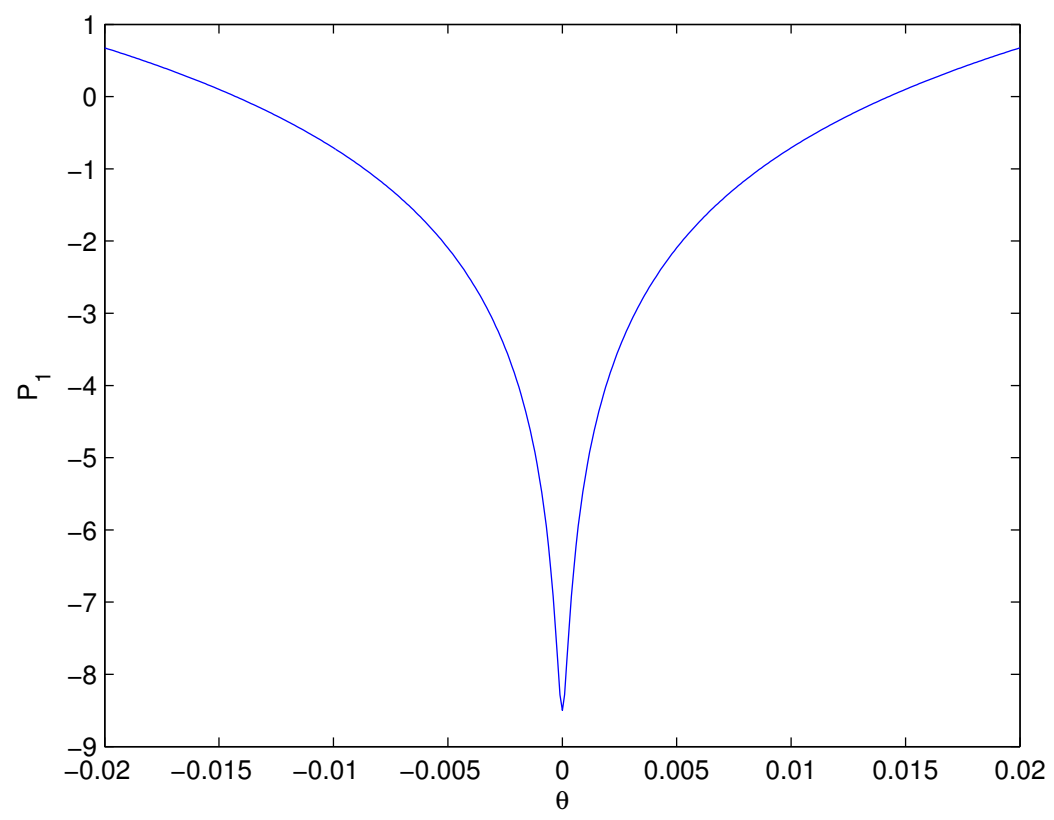

Figure 2: $P_{1}$ in the neighborhood of a false spike for a fixed $\tau$. 
By combining the expansions $(37)-(38)$ and $(40)$, it is possible to conclude that if $q(\theta) \neq 0$, there is the neighborhood of $\theta$ such that there are expansions for $\left(Q_{1}, P_{1}\right)$ of the same form as the ones for $(Q, P)$ in that neighborhood. However, at the point $\theta_{0}$, the situation is different. In fact, Equation (40) implies that

$$
P_{1}\left(\tau, \theta_{0}\right)=-P\left(\tau, \theta_{0}\right)+\ln \left[1+e^{2 P\left(\tau, \theta_{0}\right)} Q^{2}\left(\tau, \theta_{0}\right)\right] .
$$

Furthermore, the last term converges to zero exponentially due to Equation (37), Equation (38) and the fact that $q\left(\theta_{0}\right)=0$. In particular,

$$
\lim _{\tau \rightarrow \infty} P_{1 \tau}\left(\tau, \theta_{0}\right)=-v_{a}\left(\theta_{0}\right)
$$

However, for $\theta$ in the punctured neighborhood of $\theta_{0}$, we have

$$
\lim _{\tau \rightarrow \infty} P_{1 \tau}(\tau, \theta)=v_{a}(\theta)
$$

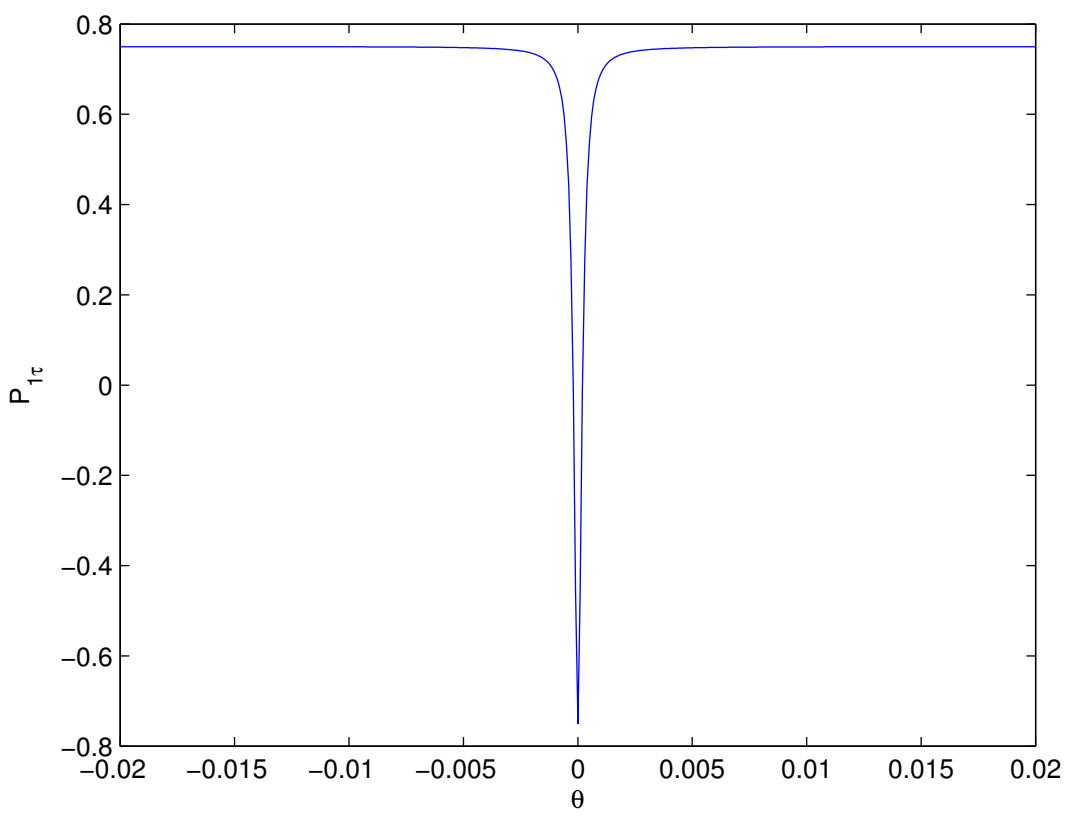

Figure 3: $P_{1 \tau}$ in the neighborhood of a false spike for a fixed $\tau$.

Due to the fact that the limit of $P_{1 \tau}$ has a discontinuity at $\theta_{0}$, the point $\theta_{0}$ is called a spike for the solution $\left(Q_{1}, P_{1}\right)$.

In order to understand this phenomenon better, it is of interest to consider the asymptotic behavior of $(Q, P)$ and $\left(Q_{1}, P_{1}\right)$ in the upper half plane model. Since $v_{a}\left(\theta_{0}\right)>0$ and $q\left(\theta_{0}\right)=0$, we have

$$
\lim _{\tau \rightarrow \infty} \phi_{R H}\left[Q\left(\tau, \theta_{0}\right), P\left(\tau, \theta_{0}\right)\right]=\lim _{\tau \rightarrow \infty}\left[Q\left(\tau, \theta_{0}\right), e^{-P\left(\tau, \theta_{0}\right)}\right]=(0,0) .
$$

In other words, the solution, at $\theta_{0}$, converges to the origin in the upper half plane. On the other hand, in the punctured neighborhood of $\theta_{0}$, the solution converges a to point on the real line different from the origin. The inversion Inv maps the origin to infinity, but it maps the points 
on the real line different from the origin to points on the real line (different from the origin). In other words, the appearance of the "spike" described above is a result of the fact that the upper half plane model of hyperbolic space has a preferred boundary point, namely infinity. In the disc model of hyperbolic space, it is not meaningful to speak of spikes of the above form. In particular,

$$
\lim _{\tau \rightarrow \infty} \mathcal{K}_{1}(\tau, \theta)=v_{a}^{2}(\theta)
$$

for all $\theta$, where $\mathcal{K}_{1}$ is the kinetic energy density associated with the solution $\left(Q_{1}, P_{1}\right)$. In other words, this limit is smooth even though the limit of $P_{1 \tau}$ is discontinuous. As a consequence of the nongeometric nature of the above spikes, we shall refer to them as "false spikes".

In Figures 1 and 2, we have plotted $Q_{1}$ and $P_{1}$, respectively, in the neighborhood of a false spike. The figures have been obtained by making a specific choice of $v_{a}, \phi, q, \psi$, ignoring $u$ and $w$ in Equations (37) - (38) and applying an inversion. They represent the solution at a fixed point in time. We have also plotted $P_{1 \tau}$ in the neighborhood of a spike in Figure 3.

\subsubsection{True spikes}

In order to give an example of a true spike, let $(Q, P)$ be a solution to $(16)-(17)$ with asymptotic expansions of the form of Equations $(37)-(38)$, where $0<v_{a}<1, q\left(\theta_{0}\right)=0$ and $q(\theta) \neq 0$ in some punctured neighborhood of $\theta_{0}$. Applying an inversion, we obtain $\left(Q_{1}, P_{1}\right)$ according to Equation (42). Applying the Gowdy to Ernst transformation produces a true spike:

$$
\left(Q_{2}, P_{2}\right)=\mathrm{GE}_{q_{0}, \tau_{0}, \theta_{0}}\left(Q_{1}, P_{1}\right)
$$

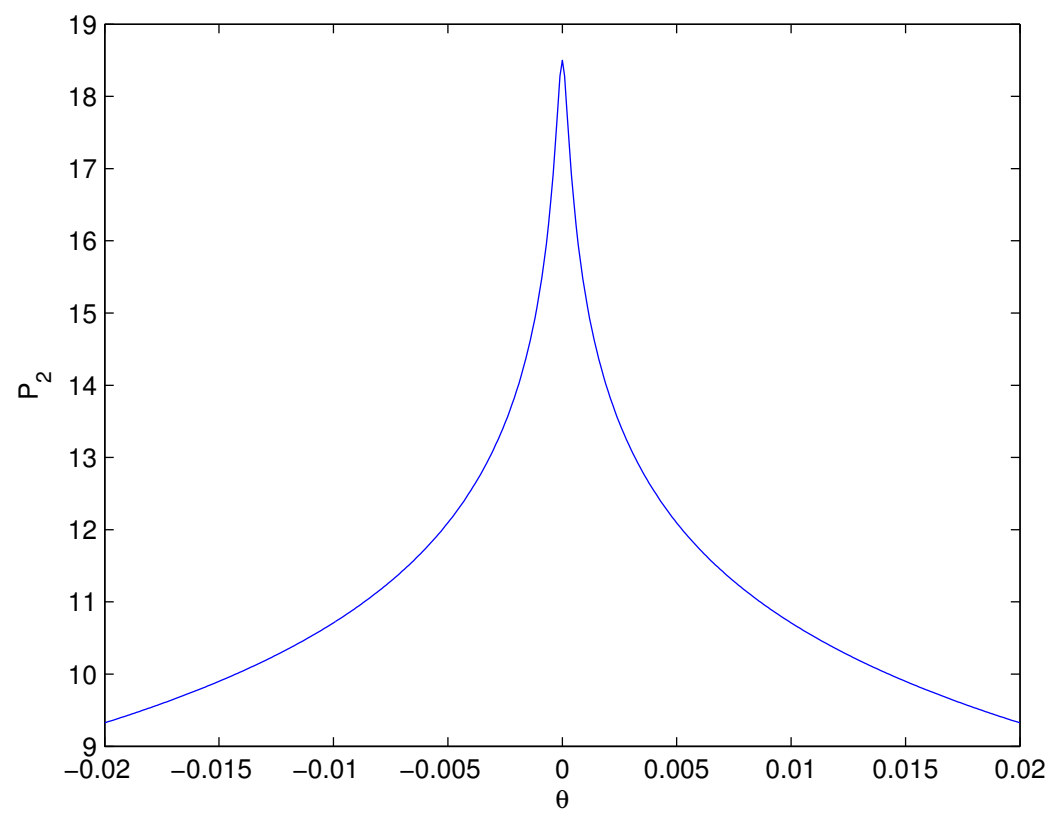

Figure 4: $P_{2}$ in the neighborhood of a true spike for a fixed $\tau$.

In Figure 4, we have plotted $P_{2}$ in the neighborhood of a true spike; we have not plotted $Q_{2}$, since it is regular in the neighborhood of the spike, even in the limit. The particular values of the 
constants $\left(q_{0}, \tau_{0}, \theta_{0}\right)$ are not of importance. Since $P_{2 \tau}=1-P_{1 \tau}$, we conclude that

$$
\lim _{\tau \rightarrow \infty} P_{2 \tau}\left(\tau, \theta_{0}\right)=1+v_{a}\left(\theta_{0}\right)
$$

due to Equation (43), and

$$
\lim _{\tau \rightarrow \infty} P_{2 \tau}(\tau, \theta)=1-v_{a}(\theta)
$$

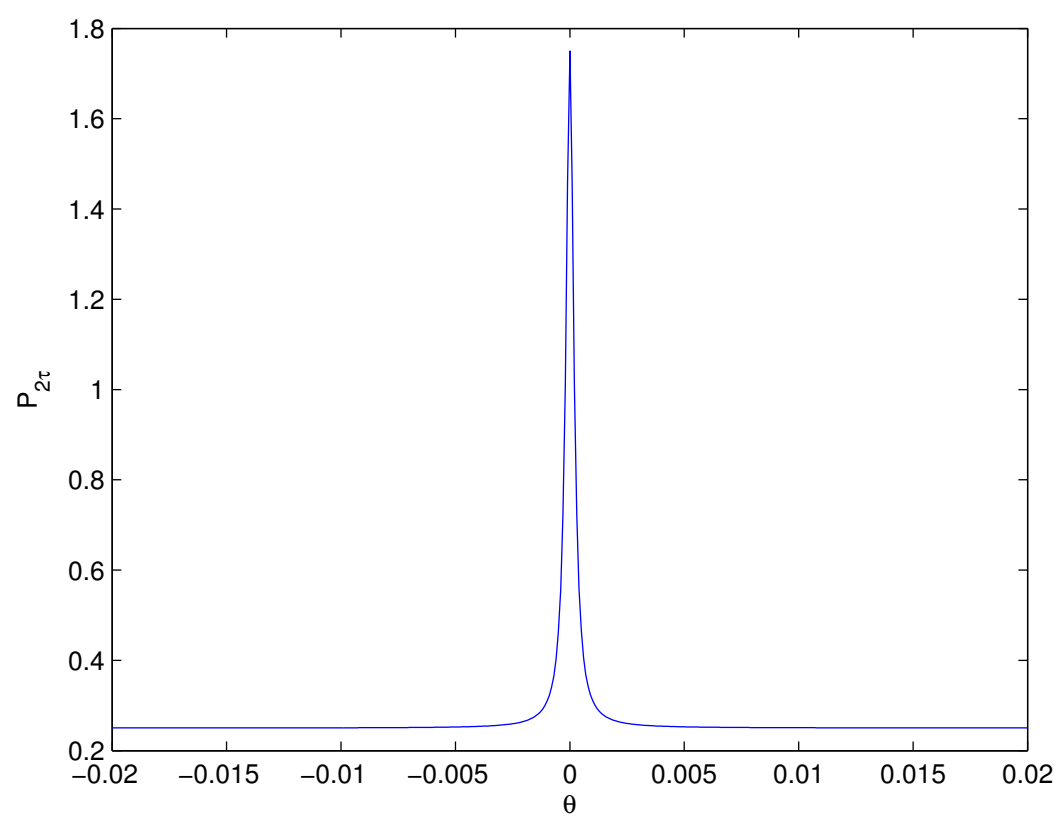

Figure 5: $P_{2 \tau}$ in the neighborhood of a true spike for a fixed $\tau$.

for $\theta$ in the punctured neighborhood of $\theta_{0}$, due to Equation (44). In Figure 5, we have plotted $P_{2 \tau}$ in the neighborhood of a true spike. In the case of a true spike, the limit of $\mathcal{K}_{2}$, the kinetic energy density associated with the solution $\left(Q_{2}, P_{2}\right)$, is discontinuous at $\theta_{0}$. Consequently, the discontinuity is a geometric feature of the solution to the wave-map equations. Furthermore, the Kretschmann scalar blows up at different rates at the tip of the spike than in the punctured neighborhood; see the bottom of $\left[71\right.$, p. 2966]. For these reasons, we shall refer to $\theta_{0}$ as a true spike associated with the solution $\left(Q_{2}, P_{2}\right)$.

\subsection{High velocity spikes}

It is possible to iterate the procedure leading to a true spike. This leads to spikes with an arbitrary high velocity; see [71, p. 2972]. In [36], numerical investigations of spikes of this type were carried out. 


\section{Asymptotic Velocity, General $T^{3}$-Gowdy}

The results we described in Section 8 and 9 consisted of constructions of solutions with certain types of asymptotics. However, considering the formulation of the strong cosmic-censorship conjecture, it is of interest to obtain conclusions given assumptions, which are phrased in terms of the initial data. The first question to ask is if there is a condition on initial data, which ensures the existence of asymptotic expansions. In [76], such a condition was established. Being phrased in terms of $L^{2}$ based energies, the condition is rather technical. However, it does have the advantage of applying to higher dimensional analogues of the equations (as opposed to much of the analysis to be described below). Other, less technical, conditions were later established $[12,74]$. Even though these results are of interest, they still only describe a part of the dynamics (as the construction of spikes, see Section 9, demonstrates). The question then arises concerning how to proceed. Considering the analysis in the polarized case, the asymptotic expansions (37)-(38) and the spikes, it is clear that the velocity $v$ plays a central role. Thus, it is natural to try to prove that it is possible to make sense of the concept of a velocity under more general circumstances.

\subsection{Existence of an asymptotic velocity}

That it is possible to define an asymptotic velocity in all generality was demonstrated in [79] (see also [12] for related results).

If there are expansions of the form (37) - (38), we have seen that $v_{a}$ can be computed according to Equation (39). As a consequence, it is of interest to ask if the limit on the right-hand side of this equation always exists. Due to [79, Corollary 6.9, p. 1009], the answer is yes. As a consequence, we are naturally led to the following definition.

Definition 4 Let $(Q, P)$ be a solution to Equations (16)-(17) and let $\theta_{0} \in S^{1}$. Then we define the asymptotic velocity at $\theta_{0}$ to be

$$
v_{\infty}\left(\theta_{0}\right)=\left[\lim _{\tau \rightarrow \infty} \mathcal{K}\left(\tau, \theta_{0}\right)\right]^{1 / 2} .
$$

\subsection{Relevance of the asymptotic velocity to the issue of curvature blow up}

Due to the definition, it is clear that $v_{\infty}$ is a geometric object from the wave-map perspective. Furthermore, it is possible to prove that if $v_{\infty}\left(\theta_{0}\right) \neq 1$, then the curvature blows up along any causal curve ending at $\theta_{0}$; see the proof of [79, Proposition 1.19, p. 989].

Note that the Gowdy metric corresponding to $P(\tau, \theta)=\tau, Q=0$ and $\lambda(\tau, \theta)=\tau$ is the flat Kasner metric. Consequently, the curvature tensor is in that case identically zero. For this solution, $v_{\infty}=1$. In particular, if $v_{\infty}\left(\theta_{0}\right)=1$, the Kretschmann scalar need not necessarily be unbounded along a causal curve ending at $\theta_{0}$.

\subsection{Interpretation of the asymptotic velocity as a rate of convergence to the boundary in hyperbolic space}

Similarly to the discussion carried out in the polarized case, the asymptotic velocity $v_{\infty}$ can be interpreted as the rate at which the solution goes to the boundary of hyperbolic space; see Equation (36). Given a solution $(Q, P)$, we shall let $\rho(\tau, \theta)$ denote the hyperbolic distance from a reference point to $[Q(\tau, \theta), P(\tau, \theta)]$ (the particular choice of reference point is not of importance). Then

$$
\lim _{\tau \rightarrow \infty} \frac{\rho\left(\tau, \theta_{0}\right)}{\tau}=v_{\infty}\left(\theta_{0}\right)
$$


see [79, Theorem 1.2, p. 982].

\subsection{Two dimensional version of the asymptotic velocity}

In the proof of strong cosmic censorship, it is of importance to note that it is meaningful to consider the velocity to be a two-dimensional object. The two-dimensional character is most easily seen by considering the solution in the disc model. Given a solution $x=(Q, P)$ to Equations (16) $-(17)$, let $z=\phi_{R D} \circ x$, where $\phi_{R D}$ is defined in Equation (24). Then the limit

$$
\lim _{\tau \rightarrow \infty}\left[\frac{z}{|z|} \frac{\rho}{\tau}\right](\tau, \theta)
$$

always exists due to [79, Lemma 6.17, p. 1011] (as an aside, it is worth noting that $\rho /|z|$ is a real analytic function from the open unit disc to the real numbers if $\rho$ is the hyperbolic distance from the origin of the unit disc to $z$; see [79, Remark 6.18, p. 1011]). It would be reasonable to call this limit, which we shall refer to as $v(\theta)$, the velocity, since it contains information not only concerning size, but also concerning direction. However, the most important aspect of this construction is that it is two-dimensional. An essential step of the proof of strong cosmic censorship is to perturb away from zero velocity. In the case of polarized Gowdy, this is not possible. However, if the velocity is a two-dimensional object, it is at least potentially possible.

\subsection{Dominant contribution to the asymptotic velocity}

It is important to note that, not only does the kinetic energy density converge pointwise, but in the limit, the only term contributing is $P_{\tau}^{2}$. In fact, the following result holds (see [79, Proposition 1.3, p. 983]):

Proposition 1 Consider a solution to Equations (16)-(17) and let $\theta_{0} \in S^{1}$. Then, either $P_{\tau}\left(\tau, \theta_{0}\right)$ converges to $v_{\infty}\left(\theta_{0}\right)$ or to $-v_{\infty}\left(\theta_{0}\right)$. If $P_{\tau}\left(\tau, \theta_{0}\right) \rightarrow-v_{\infty}\left(\theta_{0}\right)$, then $\left(Q_{1}, P_{1}\right)=\operatorname{Inv}(Q, P)$ has the property that $P_{1 \tau}\left(\tau, \theta_{0}\right) \rightarrow v_{\infty}\left(\theta_{0}\right)$. Furthermore, if $v_{\infty}\left(\theta_{0}\right)>0$, then $Q_{1}\left(\tau, \theta_{0}\right)$ converges to 0 .

Similar to what we have already seen for false spikes, see Section 9.3, we see that by applying an inversion we can always obtain a non-negative limit for $P_{\tau}$.

\subsection{Value of the asymptotic velocity as a criterion for the existence of expansions}

Not only is the asymptotic velocity a geometric quantity (from the wave-map perspective), not only can it be used as an indicator for curvature blow up, it can also be used as a criterion to determine whether asymptotic expansions exist or not. There are many results of this form, see, e.g., [12, 76, 83]. However, we shall only describe some of them, beginning with [79, Proposition 1.5, p. 984] (note that this result was essentially obtained in a previous paper [74]):

Proposition 2 Let $(Q, P)$ be a solution to Equations (16) $-(17)$ and assume $0<v_{\infty}\left(\theta_{0}\right)<1$. If $P_{\tau}\left(\tau, \theta_{0}\right)$ converges to $v_{\infty}\left(\theta_{0}\right)$, then there is an open interval $I$ containing $\theta_{0}, v_{a}, \phi, q, r \in C^{\infty}(I, \mathbb{R})$, $0<v_{a}<1$, polynomials $\Xi_{k}$ for all $k \in \mathbb{N}$ and a $T$ such that for all $\tau \geq T$

$$
\begin{aligned}
\left\|P_{\tau}(\tau, \cdot)-v_{a}\right\|_{C^{k}(I, \mathbb{R})} & \leq \Xi_{k}(\tau) e^{-\alpha \tau}, \\
\|P(\tau, \cdot)-p(\tau, \cdot)\|_{C^{k}(I, \mathbb{R})} & \leq \Xi_{k}(\tau) e^{-\alpha \tau}, \\
\left\|e^{2 p(\tau, \cdot)} Q_{\tau}(\tau, \cdot)-r\right\|_{C^{k}(I, \mathbb{R})} & \leq \Xi_{k}(\tau) e^{-\alpha \tau},
\end{aligned}
$$

Living Reviews in Relativity

http://www. livingreviews.org//rr-2010-2 


$$
\left\|e^{2 p(\tau, \cdot)}[Q(\tau, \cdot)-q]+\frac{r}{2 v_{a}}\right\|_{C^{k}(I, \mathbb{R})} \leq \Xi_{k}(\tau) e^{-\alpha \tau}
$$

where $p(\tau, \cdot)=v_{a} \cdot \tau+\phi$ and $\alpha>0$. If $P_{\tau}\left(\tau, \theta_{0}\right)$ converges to $-v_{\infty}\left(\theta_{0}\right)$, then $\operatorname{Inv}(Q, P)$ has expansions of the above form in the neighborhood of $\theta_{0}$.

It is worth noting that the above proposition proves that if $0<v_{\infty}\left(\theta_{0}\right)<1$, then $v_{\infty}$ is smooth in the neighborhood of $\theta_{0}$. In other words, knowledge concerning $v_{\infty}$ at one point can sometimes yield conclusions in the neighborhood of that point; see [79, Remark 1.6, p. 985].

Equation (48) essentially has the same content as Equation (38). In order to see this, define the object inside the norm on the left-hand side of Equation (48) to be $\tilde{w}$. Then

$$
Q=q+e^{-2 p}\left[-\frac{r}{2 v_{a}}+\tilde{w}\right]
$$

Using the above expansions and equations, expressions for the higher-order time derivatives can be derived. 


\section{The Generic Set, General $T^{3}$-Gowdy}

There are general results concerning the asymptotic behavior in the direction of the singularity for $T^{3}$-Gowdy spacetimes; there is, e.g., an open and dense subset $\mathcal{E}$ of the circle such that there are smooth expansions on $\mathcal{E}$; see [12, Theorem 1.3, p. 1018] and [79, Proposition 1.9, p. 985]. However, the results of $[54,68,71]$ show that the asymptotic behavior of solutions is in general very complicated; there are, e.g., solutions with an infinite number of true spikes. On the other hand, much of the complicated behavior can be expected to be unstable, i.e., nongeneric. Thus, since the strong cosmic-censorship conjecture is only a statement concerning generic solutions, it is natural to try to find a set of solutions whose asymptotics are generic but less complicated than those of general solutions. The purpose of the present section is to define one generic set of initial data. Since the concepts nondegenerate true and false spikes play a central role, let us begin by defining them.

\subsection{Nondegenerate true spikes}

The definition of a nondegenerate true spike proceeds by running the construction of a true spike backwards. In other words, we start with a solution $(Q, P)$ such that $1<v_{\infty}\left(\theta_{0}\right)<2$ and such that $P_{\tau}\left(\tau, \theta_{0}\right) \rightarrow v_{\infty}\left(\theta_{0}\right)$. Letting $\left(Q_{1}, P_{1}\right)=\mathrm{GE}_{q_{0}, \tau_{0}, \theta_{0}}(Q, P)$, we see, by Equation (41), that $P_{1 \tau}\left(\tau, \theta_{0}\right) \rightarrow 1-v_{\infty}\left(\theta_{0}\right)<0$. Let $\left(Q_{2}, P_{2}\right)=\operatorname{Inv}\left(Q_{1}, P_{1}\right)$. Then, due to Proposition 1 , $P_{2 \tau}\left(\tau, \theta_{0}\right) \rightarrow v_{\infty}\left(\theta_{0}\right)-1$ and $Q_{2}\left(\tau, \theta_{0}\right) \rightarrow 0$. Finally, Proposition 2 applies to $\left(Q_{2}, P_{2}\right)$ so that there are smooth expansions in the neighborhood $I$ of $\theta_{0}$. In particular, there is a smooth function $q_{2}$ such that $Q_{2}$ converges to $q_{2}$ with respect to any $C^{k}$-norm. Moreover, it is important to note that $q_{2}\left(\theta_{0}\right)=0$. We are naturally led to [79, Definition 1.12, p. 987]:

Definition 5 Consider a solution $(Q, P)$ to Equations (16)-(17). Assume $1<v_{\infty}\left(\theta_{0}\right)<2$ for some $\theta_{0} \in S^{1}$ and that

$$
\lim _{\tau \rightarrow \infty} P_{\tau}\left(\tau, \theta_{0}\right)=v_{\infty}\left(\theta_{0}\right)
$$

Let $\left(Q_{2}, P_{2}\right)=\operatorname{Inv} \circ \mathrm{GE}_{q_{0}, \tau_{0}, \theta_{0}}(Q, P)$. By the observations made prior to the definition, $\left(Q_{2}, P_{2}\right)$ has smooth expansions in the neighborhood I of $\theta_{0}$. In particular $Q_{2}$ converges to a smooth function $q_{2}$ in $I$ and the convergence is exponential in any $C^{k}$-norm. We call $\theta_{0}$ a non-degenerate true spike if $\partial_{\theta} q_{2}\left(\theta_{0}\right) \neq 0$.

The choice of $q_{0}, \tau_{0}, \theta_{0}$ is unimportant. Note that nondegenerate true spikes have punctured neighborhoods with normal expansions.

\subsection{Nondegenerate false spikes}

Let us recall the definition of a nondegenerate false spike, [79, Definition 1.11, p. 986]:

Definition 6 Consider a solution $(Q, P)$ to Equations (16)-(17). Assume $0<v_{\infty}\left(\theta_{0}\right)<1$ for some $\theta_{0} \in S^{1}$ and that

$$
\lim _{\tau \rightarrow \infty} P_{\tau}\left(\tau, \theta_{0}\right)=-v_{\infty}\left(\theta_{0}\right)
$$

Let $\left(Q_{1}, P_{1}\right)=\operatorname{Inv}(Q, P)$. By Proposition 2, we get the conclusion that $\left(Q_{1}, P_{1}\right)$ has smooth expansions in a neighborhood $I$ of $\theta_{0}$. In particular, $Q_{1}$ converges to a smooth function $q_{1}$ in $I$, and the convergence is exponential in any $C^{k}$-norm. By Proposition 1, $q_{1}\left(\theta_{0}\right)=0$. We call $\theta_{0}$ a nondegenerate false spike if $\partial_{\theta} q_{1}\left(\theta_{0}\right) \neq 0$.

Note that nondegenerate false spikes have punctured neighborhoods with normal expansions. 


\subsection{The generic set, definition}

We are now in a position to give the definition of what, in the end, will turn out to be the generic set, [79, Definition 1.14, p. 988]:

Definition 7 Let $\mathcal{G}_{l, m}$ be the set of smooth solutions $(Q, P)$ to Equations (16)-(17) on $\mathbb{R} \times S^{1}$ with $l$ nondegenerate true spikes $\theta_{1}, \ldots, \theta_{l}$ and $m$ nondegenerate false spikes $\theta_{1}^{\prime}, \ldots, \theta_{m}^{\prime}$ such that

$$
\lim _{\tau \rightarrow \infty} P_{\tau}(\tau, \theta)=v_{\infty}(\theta)
$$

for all $\theta \notin\left\{\theta_{1}^{\prime}, \ldots, \theta_{m}^{\prime}\right\}$ and $0<v_{\infty}(\theta)<1$ for all $\theta \notin\left\{\theta_{1}, \ldots, \theta_{l}\right\}$. Let $\mathcal{G}_{l, m, c}$ be the set of $(Q, P) \in \mathcal{G}_{l, m}$ such that

$$
\int_{S^{1}}\left(P_{\tau} P_{\theta}+e^{2 P} Q_{\tau} Q_{\theta}\right) d \theta=0
$$

Finally

$$
\mathcal{G}=\bigcup_{l=0}^{\infty} \bigcup_{m=0}^{\infty} \mathcal{G}_{l, m}, \quad \mathcal{G}_{c}=\bigcup_{l=0}^{\infty} \bigcup_{m=0}^{\infty} \mathcal{G}_{l, m, c} .
$$

\subsection{Verification of genericity, openness}

If it is possible to prove that $\mathcal{G}$ is open and dense, it is justified to call it a generic set. A first step in this direction is given by [79, Proposition 1.15, p. 988]:

Proposition $3 \mathcal{G}_{l, m}$ is open in the $C^{2} \times C^{1}$-topology on initial data and $\mathcal{G}_{l, m, c}$ is open in the $C^{2} \times C^{1}$-topology on the subset of initial data satisfying Equation (49).

It is of interest to note that the topology can be weakened somewhat if the only information of interest concerning the asymptotics is that the asymptotic velocity is different from 1 . In fact, [79, Proposition 1.16, p. 988] states:

Proposition 4 Given $z \in \mathcal{G}_{l, m}$, there is an open neighborhood of the initial data for $z$ in the $C^{1} \times C^{0}$ topology such that for each corresponding solution $\hat{z}, v_{\infty}[\hat{z}](\theta) \in(0,1) \cup(1,2)$ for all $\theta \in S^{1}$.

Recall that an asymptotic velocity different from 1 implies curvature blow up.

\subsection{Verification of genericity, density}

Finally, [83, Theorem 2, p. 1190] yields the conclusion that $\mathcal{G}$ is dense:

Theorem $3 \mathcal{G}$ and $\mathcal{G}_{c}$ are dense in $\mathcal{S}_{p}$ and $\mathcal{S}_{p, c}$, respectively, with respect to the $C^{\infty}$-topology on initial data.

Here $\mathcal{S}_{p}$ and $\mathcal{S}_{p, c}$ are defined in [83, Definition 1, p. 1188]:

Definition 8 Let $\mathcal{S}_{p}$ denote the set of smooth solutions to Equations (16)-(17) on $\mathbb{R} \times S^{1}$, and let $\mathcal{S}_{p, c}$ denote the subset of $\mathcal{S}_{p}$ obeying

$$
\int_{S^{1}}\left(P_{\tau} P_{\theta}+e^{2 P} Q_{\tau} Q_{\theta}\right) d \theta=0
$$




\section{Expanding Direction, Polarized Gowdy}

Since there is only an expanding direction in the case when spatial topology is $T^{3}$, the current section is restricted to considerations of that case. For our purposes, it would be sufficient to describe the results in the general case, since that would yield all the information needed concerning the polarized case as well. However, for historical reasons, and since results have been obtained in the polarized case that are as yet unavailable in the general case, we shall describe both.

Even though it is of no immediate relevance to the question of strong cosmic censorship, let us note that there is a general picture concerning the future asymptotic behavior of vacuum solutions to Einstein's equations; see [1,33] and references cited therein. In particular, a strong connection between the future asymptotics and spatial topology is conjectured to exist. In [80] it was confirmed that vacuum $T^{3}$-Gowdy fits into the general picture.

\subsection{Asymptotic behavior}

That the polarized vacuum $T^{3}$-Gowdy spacetimes are future causally geodesically complete was announced in [21]; see [21, Step 3, p. 1677]. However, we shall here follow the presentation of [52]. The relevant equation to study is Equation (29). However, in the study of the expanding direction, it is convenient to change the time coordinate to the original areal time $t$. The equation then becomes

$$
P_{t t}+\frac{1}{t} P_{t}-P_{\theta \theta}=0
$$

Since the equation is linear and the coefficients do not depend on the spatial coordinate, it is clear that the spatial average of $P$,

$$
\langle P\rangle=\frac{1}{2 \pi} \int_{S^{1}} P(\cdot, \theta) d \theta
$$

solves the same equation. Furthermore, it is clear that there are constants $a$ and $b$ such that

$$
\langle P\rangle=a \ln t+b .
$$

It is of interest to know what the asymptotic behavior of the remainder is. It turns out that there is a solution $\nu$ to the ordinary wave equation, i.e.,

$$
\nu_{t t}-\nu_{\theta \theta}=0
$$

with zero average, i.e.,

$$
\int_{S^{1}} \nu(t, \theta)=0
$$

for all $t>0$, and a function $\psi$, the average of which is also zero, such that

$$
P(t, \theta)=a \ln t+b+t^{-1 / 2} \nu(t, \theta)+\psi(t, \theta) .
$$

Furthermore, $\psi$ and its first derivatives decay as $t^{-3 / 2}$ and the division of Equation (52) is unique; see [52, Corollary 11, p. 183] (note that the statement of this result is also to be found in [21, (7a), p. 1675]). In short, given a solution $P$, there are $a, b$ and $\nu$ as above (and then $\psi$ is uniquely determined). It is of interest to ask if it is possible to go in the other direction. In other words, given $a, b$ and $\nu$ as above, is there a solution with the above form of asymptotics. The answer to this question is yes; see [78, Proposition 1, p. 1649]. In other words, $a, b$ and $\nu$, with properties as above can be considered to be data at the moment of infinite expansion. Using the above information, it is possible to prove that polarized vacuum $T^{3}$-Gowdy spacetimes are future causally-geodesically complete; see [52, Corollary 21, p. 190]. 


\subsection{Comparison with spatially homogeneous solutions}

Due to Equation (52), it seems natural to say that the solution tends to a spatially homogeneous solution to the equations. However, this conclusion is strongly dependent on the norm chosen for measuring deviations from spatial homogeneity. In fact, when comparing the time derivatives, the situation is completely different;

$$
\frac{P_{t}-\left\langle P_{t}\right\rangle}{\left\langle P_{t}\right\rangle}
$$

is generically unbounded to the future. In other words, with respect to this measure, the solution is far from being spatially homogeneous. 


\section{Expanding Direction, The General Case}

Clearly, the situation is more complicated in the nonpolarized case. Consequently, it is natural to start by asking questions concerning the rough behavior of solutions. It is also natural to carry out numerical simulations. This was done in [8], a paper, which played an important role in the further development of the subject. In the polarized case, the difference between the solution and its spatial average converges to zero. Does the same phenomenon occur in the general case? This is perhaps too complicated a question to start with, but it is of interest to know how the spatial variation of the solution evolves with time. In order to define what is meant by the spatial variation, recall that Equations (11)-(12) can be viewed as wave-map equations in which the metric of the target is given by Equation (20). Furthermore, at each point in time, the solution defines a loop in hyperbolic space. The natural definition of the spatial variation of the solution at one point in time is the length of this loop with respect to the hyperbolic metric (20). In other words, it is natural to ask how

$$
\ell=\int_{S^{1}} \sqrt{P_{\theta}^{2}+e^{2 P} Q_{\theta}^{2}} d \theta
$$

evolves with time. In the case of polarized Gowdy, we know that

$$
P_{\theta}=t^{-1 / 2} \nu_{\theta}+O\left(t^{-1}\right)
$$

asymptotically; see Equation (52) and the adjacent text. Consequently, $\ell=O\left(t^{-1 / 2}\right)$ in that case, but there is no better estimate.

\subsection{Energy decay}

In order to analyze the general case, it is of interest to consider the energy

$$
H=\frac{1}{2} \int_{S^{1}}\left[P_{t}^{2}+P_{\theta}^{2}+e^{2 P}\left(Q_{t}^{2}+Q_{\theta}^{2}\right)\right] d \theta .
$$

The numerical studies of Berger and Moncrief [8] indicate that this quantity should decay as $1 / t$. Using Hölder's inequality, this would imply that $\ell=O\left(t^{-1 / 2}\right)$. That this is the behavior, which actually occurs was later established in [75, Theorem 1.1, p. 660]:

Theorem 4 Consider a solution to Equations (11)-(12). Then there is a $T \geq 1$ and a $K$ such that for all $t \geq T$, the energy $H$ defined by Equation (53) satisfies

$$
H(t) \leq \frac{K}{t}
$$

In fact, this result can be improved somewhat to [75, Theorem 1.6, p. 664]:

Theorem 5 Consider a solution to Equations (11)-(12). Then if $H$ is given by Equation (53), there is a K, a T>0 and a constant $c_{H}$ such that

$$
\left|t H(t)-c_{H}\right| \leq \frac{K}{t}
$$

for all $t \geq T$. Furthermore, if $c_{H}$ is zero, the solution is independent of $\theta$, and in that case, $t^{2} H(t)$ is constant.

Note that, in some respects, this result leads to conclusions that, on an intuitive level, are somewhat contradictory. First, since $H$ converges to zero, it is clear that the spatial variation of the solution, i.e., $\ell$, converges to zero. Consequently, it seems natural to expect the solution to behave as a 
spatially homogeneous solution to the equations. Thus, consider a non-spatially-homogeneous solution and a spatially homogeneous solution, which is supposed to approximate it, and let $H_{\text {sol }}$ and $H_{\text {hom }}$ denote the corresponding energies. Then, due to Theorem 5 ,

$$
\lim _{t \rightarrow \infty} \frac{H_{\mathrm{sol}}-H_{\mathrm{hom}}}{H_{\mathrm{hom}}}=\infty, \quad \lim _{t \rightarrow \infty} \frac{H_{\mathrm{sol}}-H_{\mathrm{hom}}}{H_{\mathrm{sol}}}=1,
$$

even though both limits should be zero if the solution is well approximated by a spatially homogeneous solution.

\subsection{Proof of decay of the energy}

Let us briefly review the idea behind the proof of Theorem 4 [75, Section 4, pp. 668-670]. We shall do so by considering a simple example. Consider, in the polarized case, a solution of the form $P(t, \theta)=x(t) \cos \theta$. With this ansatz, Equation (51) is equivalent to

$$
\ddot{x}+\frac{1}{t} \dot{x}+x=0 .
$$

\subsubsection{Toy model}

Let us simplify the above equation further and consider

$$
\ddot{x}+2 a \dot{x}+b^{2} x=0,
$$

where $a$ and $b$ are constants and $a>0$ and $b^{2}>a^{2}$. Clearly, this equation is of no interest in itself; there is no need to develop methods for analyzing an equation, which can be solved explicitly. However, considering this equation from a different perspective might lead to the development of methods that can be used in a more general situation. Since energies have turned out to be very useful in the analysis of systems of nonlinear wave equations, let us consider

$$
H=\frac{1}{2}\left(\dot{x}^{2}+b^{2} x^{2}\right)
$$

We know that this quantity decreases exponentially as $e^{-2 a t}$. Let us try to prove this statement without explicitly solving the equation. A natural first step is to differentiate:

$$
\frac{d H}{d t}=-2 a \dot{x}^{2} .
$$

Clearly, $H$ decreases. However, this is only a qualitative statement. In order to obtain a quantitative statement, let us introduce

$$
\Gamma=a x \dot{x} .
$$

We shall refer to this quantity as a correction term. It is important to note that this object has the following two properties. First

$$
|\Gamma|=\left|\frac{a}{b}\right||b x \dot{x}| \leq\left|\frac{a}{b}\right| \frac{1}{2}\left(\dot{x}^{2}+b^{2} x^{2}\right)=\left|\frac{a}{b}\right| H .
$$

As a consequence, there are constants $c_{1}, c_{2}>0$ such that

$$
c_{1} H \leq H+\Gamma \leq c_{2} H
$$

recall that $|a / b|<1$. Second,

$$
\frac{d(H+\Gamma)}{d t}=-2 a(H+\Gamma) .
$$

Combining these two properties, we obtain $H \leq K \exp (-2 a t)$. This estimate is optimal. 


\subsubsection{Polarized case}

In the case of the polarized Gowdy equation (51) it is possible to carry out a similar argument. In fact, the correction

$$
\Gamma=\frac{1}{2 t} \int_{S^{1}}(P-\langle P\rangle) P_{t} d \theta
$$

can be used to prove that $H \leq K / t$ [75, Section 4, pp. 668-670] for the details.

\subsubsection{General case}

Ideas similar to the above can be used in the general Gowdy case, though there are additional complications. Due to the nonlinear character of the problem, it is necessary to divide the proof of the decay of the energy into two parts. The first part involves proving that if the energy is small initially, then the energy decays as $1 / t$. The second step consists of proving that the energy converges to zero. The small data result is, just as above, based on the introduction of a certain correction $\Gamma$ with the properties that

$$
|\Gamma| \leq \frac{K}{t} H
$$

and that

$$
\frac{d(H+\Gamma)}{d t} \leq-\frac{1}{t}(H+\Gamma)-\frac{1}{t} \Gamma+\frac{K}{t} H^{3 / 2}
$$

Combining these two inequalities, it is possible to conclude that $H=O\left(t^{-1}\right)$, assuming $H$ to be small enough initially.

One way to take the step from small data to large data is to prove that $H$ converges to zero. What is known a priori is that

$$
\frac{d H}{d t}=-\frac{1}{t} \int_{S^{1}}\left(P_{t}^{2}+e^{2 P} Q_{t}^{2}\right) d \theta .
$$

Just as before, this implies that $H$ decays, but not that $H$ converges to zero. However, it does prove that the right-hand side of Equation (57) is integrable. This information might not seem so useful. However, if it were possible to prove the integrability of $H / t$ to the future, we would be allowed to conclude that $H$ converges to zero (recall that $H$ is monotonically decreasing). This would then finish the result. In order to take the step from integrability of the right-hand side of (57) to the integrability of $H / t$, we need to prove that

$$
\int_{t_{0}}^{t} \frac{1}{s} \int_{S^{1}}\left(P_{t}^{2}-P_{\theta}^{2}\right) d \theta d s, \quad \int_{t_{0}}^{t} \frac{1}{s} \int_{S^{1}} e^{2 P}\left(Q_{t}^{2}-Q_{\theta}^{2}\right) d \theta d s
$$

are bounded to the future. Note that these expressions are far from arbitrary. It is natural to integrate by parts twice and to use the equations (it is of some interest to note that the order in which one considers the two expressions is very important). Doing so leads to the desired conclusion; see [75] for the details.

It is of interest to note that the results concerning the decay rate can be generalized to a larger class of spacetimes [81].

\subsection{Asymptotic ODE behavior}

In the case of polarized Gowdy, the solution asymptotically behaves like a spatially homogeneous solution to the same equation (at least in some respects). Is it possible to prove something similar 
in the general case? In the polarized case, the averaged equation is equivalent to the statement that an integral is conserved; there is a constant $K$ such that

$$
\int_{S^{1}} t P_{t} d \theta=K
$$

Furthermore, this equation can be interpreted as an ODE for $\langle P\rangle$. Are there analogous conserved quantities in the general case?

\subsubsection{Conserved quantities}

As has been noted before, we have the conserved quantities $A, B$ and $C$ given by Equations(25)(27). We shall also use the notation $\alpha=A / 2 \pi, \beta=B / 2 \pi, \gamma=C / 2 \pi$ and

$$
\delta=\frac{\sqrt{\left|\alpha^{2}+4 \beta \gamma\right|}}{2} .
$$

As was mentioned in Section 6.4, applying an isometry of hyperbolic space to a solution yields a new solution. It is of interest to know what the corresponding change in the conserved quantities $A, B$ and $C$ is. It turns out that the quantity $A^{2}+4 B C$ remains unchanged; see the proof of [75, Lemma 8.2 , p. 681]. Note that in the spatially homogeneous case,

$$
\alpha^{2}+4 \beta \gamma=4 t^{2}\left(P_{t}^{2}+e^{2 P} Q_{t}^{2}\right) .
$$

In other words, $A^{2}+4 B C \geq 0$ for spatially homogeneous solutions. However, in the inhomogeneous case, $A^{2}+4 B C$ can take any value; see $[75,(6.9)-(6.10)$, p. 676$]$ as well as the adjacent text. Consequently, it seems unlikely that it should be possible to approximate solutions such that $A^{2}+4 B C$ is negative by spatially homogeneous solutions.

In practice, it is often convenient to apply an isometry to a solution so that the conserved quantities become as simple as possible. This is achieved in [75, Lemma 8.2, p. 681]:

Lemma 1 Consider a solution to Equations (11)-(12). If $A^{2}+4 B C>0$, there is an isometry such that if $A_{1}, B_{1}$ and $C_{1}$ are the constants of the transformed solution, then $A_{1}=-\sqrt{A^{2}+4 B C}$ and $B_{1}=C_{1}=0$. If $A^{2}+4 B C=0$, there is an isometry such that the constants of the transformed solution are $A_{1}=B_{1}=0$ and $C_{1}=4 \pi$ or $C_{1}=0$.

Analyzing the asymptotic behavior of the transformed solution and then transforming back is often more convenient than analyzing the original solution.

\subsubsection{Interpreting the conserved quantities as ODEs for the averages}

Returning to the question of the asymptotics, we wish to interpret the conserved quantities as ODEs for $\langle P\rangle$ and $\langle Q\rangle$. Due to [75, Lemma 8.1, p. 680], we have the following result:

Lemma 2 Consider a solution to Equations (11)-(12). Then

$$
\begin{aligned}
t\left\langle P_{t}\right\rangle & =\beta\langle Q\rangle-\frac{\alpha}{2}+\frac{1}{2 \pi} \int_{S^{1}} t e^{2 P}(Q-\langle Q\rangle) Q_{t} d \theta, \\
t e^{\langle P\rangle}\left\langle Q_{t}\right\rangle & =\beta e^{-\langle P\rangle}-\frac{1}{2 \pi} e^{\langle P\rangle} \int_{S^{1}}\left(e^{2 P-2\langle P\rangle}-1\right) t Q_{t} d \theta, \\
t\left\langle Q_{t}\right\rangle & =\gamma+\alpha\langle Q\rangle-\beta\langle Q\rangle^{2}+\frac{t}{\pi} \int_{S^{1}}(\langle Q\rangle-Q) P_{t} d \theta+\frac{t}{2 \pi} \int_{S^{1}} e^{2 P} Q_{t}(Q-\langle Q\rangle)^{2} d \theta .
\end{aligned}
$$


Naively, it would seem natural to consider the integral expressions to be error terms and to interpret what remains as ODEs for the averages. On the other hand, it would then seem that we have too many equations. In the end, the situation turns out to be somewhat more complicated; see below.

Among other things, Equations (61) - (63) imply the existence of a constant $K$ such that

$$
\left\langle P_{t}\right\rangle^{2}+e^{2\langle P\rangle}\left\langle Q_{t}\right\rangle^{2} \leq \frac{K}{t^{2}}
$$

Note that this result is similar to the one obtained in the polarized case. Moreover, just as in the polarized case, it is a rather surprising result. Due to Theorem 5 and 57 , the best estimate that can be obtained for

$$
\int_{S^{1}}\left(P_{t}^{2}+e^{2 P} Q_{t}^{2}\right) d \theta
$$

is that it decays as $t^{-1}$. In other words, first taking the average and then taking the square leads to decay of the form $t^{-2}$. First taking the square and then taking the average leads to decay of the form $t^{-1}$. This behavior reflects the same sort of asymptotics as those characterized by Equation (52).

Naively estimating the integral on the right-hand side of Equation (61) leads to the conclusion that it is bounded but no more. Consequently, it seems unreasonable to think of this term as an error term. On the other hand, integrating with respect to time might lead to an improvement. In fact, since $\left\langle Q_{t}\right\rangle$ decays very quickly, see Equation (64), replacing $Q_{t}$ with $\left\langle Q_{t}\right\rangle$ in Equation (61) leads to a term, which tends to zero. Consequently, we can replace $Q_{t}$ by $Q_{t}-\left\langle Q_{t}\right\rangle$ in (61) with a small error. Integrating Equation (61) and using such ideas leads to [75, Lemma 8.9, p. 685]:

Lemma 3 Consider a solution to Equations (11)-(12). Then if $t>t_{0} \geq 1$

$$
\int_{t_{0}}^{t}\left[\left\langle P_{t}\right\rangle-\frac{\beta}{s}\langle Q\rangle+\frac{\alpha}{2 s}\right] d s=O\left(t_{0}^{-1 / 2}\right) .
$$

Note that in the case of $B=0$, this result gives detailed information concerning the asymptotics of $\langle P\rangle$; see [75, Corollary 8.10, p. 685]:

Corollary 1 Consider a solution to Equations (11)-(12). If $B=0$ there is a constant $c_{P}$ and a $T>0$ such that

$$
\langle P\rangle+\frac{\alpha}{2} \ln t-c_{P}=O\left(t^{-1 / 2}\right)
$$

for all $t \geq T$.

Clearly, Lemma 3 yields important information concerning the asymptotics. Is it possible to apply similar ideas to Equations (62) and (63)? It turns out to be necessary to combine both equations in order to obtain a single equation for $\langle Q\rangle$. The problem is the last term in Equation (62) and the second to last term in Equation (63). However, combining partial integrations, Taylor expansions in the last term in Equation (62) with various estimates, such as Equation (64), leads to [75, Lemma 8.8, p. 684]:

Lemma 4 Consider a solution to Equations (11)-(12) and let $f \in C^{\infty}\left(\mathbb{R}_{+}, \mathbb{R}\right)$ satisfy

$$
\left|e^{-\langle P\rangle} f\right| \leq K \text { and }\left|e^{-\langle P\rangle} f_{t}\right| \leq \frac{K}{t^{1 / 2}}
$$

for $t \geq T$. Then, if $t \geq t_{0} \geq T$,

$$
\int_{t_{0}}^{t} f\left[2\left\langle Q_{t}\right\rangle-\frac{\gamma}{s}-\frac{\alpha}{s}\langle Q\rangle+\frac{\beta}{s}\langle Q\rangle^{2}-\frac{\beta}{s} e^{-2\langle P\rangle}\right] d s=O\left(t_{0}^{-1 / 2}\right) .
$$


In the case of $B=0$ it is convenient to apply this result with $f=t^{-\alpha / 2}$. This leads to [75, Proposition 8.11, p. 685]:

Proposition 5 Consider a solution to Equations (11)-(12). If $B=0$, then there is a constant $c_{Q}$ and $a T>0$ such that for $t \geq T$,

$$
\left|e^{\langle P\rangle}\left(\langle Q\rangle+\frac{\gamma}{\alpha}\right)-c_{Q}\right| \leq K t^{-1 / 2}
$$

if $\alpha \neq 0$ and

$$
\left|\langle Q\rangle-\frac{\gamma}{2} \ln t-c_{Q}\right| \leq K t^{-1 / 2}
$$

if $\alpha=0$.

In case of $B=0$, we consequently have detailed information concerning the asymptotic behavior of $\langle Q\rangle$ as well. Furthermore, as was observed earlier, given a solution with the property that $A^{2}+4 B C \geq 0$, there is an isometry of hyperbolic space such that the transformed solution is such that the corresponding $B$ equals zero. Thus, the only case that remains to be analyzed is $A^{2}+4 B C<0$. This case is more complicated (but more interesting). We shall therefore omit a description of the analysis.

\subsection{Geometric interpretation of the asymptotics}

The analysis of the asymptotics in the various cases leads to the following conclusion $[75$, Theorem 1.2 , p. 661]:

Theorem 6 Consider a solution to Equations (11)-(12). Let $\mathbf{x}=(x, y)=\left(Q, e^{-P}\right)$ and let $d_{H}$ be the metric induced by the Riemannian metric $g_{H}$ given by Equation (22). Then there is a K, a $T>0$ and a curve $\Gamma$ such that

$$
d_{H}(\mathbf{x}(t, \theta), \Gamma) \leq K t^{-1 / 2}
$$

for all $t \geq T$. The possibilities for $\Gamma$ are as follows.

- If all the constants $A, B$ and $C$ are zero, $\Gamma$ is a point.

- If $A^{2}+4 B C=0$, but the constants are not all zero, $\Gamma$ is either a horocycle (i.e., a circle touching the boundary) or a curve $y=$ constant.

- If $A^{2}+4 B C>0, \Gamma$ is either a circle intersecting the boundary transversally or a straight line intersecting the boundary transversally.

- If $A^{2}+4 B C<0, \Gamma$ is a circle inside the upper half plane.

Furthermore, it is possible to describe in detail the behavior of the solutions along the circles; see [75, Theorem 1.3-1.5, pp. 662-664] as well as [75, Figure 1.1-1.3, pp. 663-664]. In fact, the solution tends to the boundary along the circle when $A^{2}+4 B C \geq 0$ and $A, B$ and $C$ are not all equal to zero. In the case of $A^{2}+4 B C<0$, the solution oscillates around the circle forever and is asymptotically periodic with respect to a logarithmic time coordinate. 


\subsection{Concluding remarks}

It is of interest to note that in the case of $A^{2}+4 B C<0$, the spatial variation dies out and the solution behaves like a solution to an ODE asymptotically. On the other hand, the ODE of which it is approximately a solution is not the ODE, which is obtained by dropping the spatial derivatives in the original equation.

Furthermore, in the polarized case, the integrand appearing on the left-hand side of Equation (58) is unbounded as $t \rightarrow \infty$. In fact, the best bound for the integrand is $C t^{1 / 2}$ due to Equation (52). On the other hand, the integral is conserved. Moreover, since this conserved quantity determines the overall behavior of the solution, it is clear that the problem of analyzing the asymptotics numerically is not trivial. The same phenomenon appears in the nonpolarized case. However, it is of interest to note that the reason why the mathematical analysis is possible is in part due to the difference in decay rates between $\left\langle P_{t}\right\rangle^{2}$ and

$$
\int_{S^{1}} P_{t}^{2} d \theta
$$

\subsection{Geodesic completeness}

The analysis described above concerned only the functions and their averages. In particular, no estimates for the derivatives in the sup norm were derived. However, in order to prove future causal geodesic completeness, it is of interest to have such estimates. According to [75, Proposition 1.8, p. 665]:

Proposition 6 Consider a solution to Equations (11)-(12). Then

$$
\left\|P_{t}\right\|_{C\left(S^{1}, \mathbb{R}\right)}+\left\|P_{\theta}\right\|_{C\left(S^{1}, \mathbb{R}\right)}+\left\|e^{P} Q_{t}\right\|_{C\left(S^{1}, \mathbb{R}\right)}+\left\|e^{P} Q_{\theta}\right\|_{C\left(S^{1}, \mathbb{R}\right)} \leq K t^{-1 / 2} .
$$

Using this estimate, it is then possible to prove future causal geodesic completeness; see [75, Theorem 1.9, p. 665]. 


\section{Strong Cosmic Censorship in Gowdy Spacetimes}

The results concerning strong cosmic censorship that exist concern the polarized case as well as the general $T^{3}$-case. Since the methods in the two cases are different, it is natural to divide the exposition accordingly.

\subsection{The polarized case}

As has already been mentioned, the proof of strong cosmic censorship in the polarized Gowdy case proceeds via Conjecture 2. In other words, via a proof of the fact that for generic initial data, the Kretschmann scalar is unbounded in the incomplete directions of causal geodesics. Future causal geodesic completeness in the polarized $T^{3}$-Gowdy case was announced in [21] and proven in [52, Corollary 21, p. 190]. Thus the main problem is that of proving that the curvature blows up at the singularities. This is achieved in two steps in [21]. First, the existence of a diffeomorphism between asymptotic data (i.e., $v$ and $\phi$ in Equation (35)) and ordinary initial data is demonstrated; see [21, p. 1675]. Second, using the observations made concerning curvature blow up in Section 7.4, it can be shown that there is an open and dense subset of the set of asymptotic data such that the curvature of the corresponding solutions blows up everywhere on the singularity. Let us state the result as given in $[21$, p. 1673$]$ :

Theorem 7 (Strong cosmic censorship for polarized Gowdy spacetimes) Let $\Sigma^{3}=T^{3}$, $S^{3}$, or $S^{2} \times S^{1}$, and let $\mathcal{P}\left(\Sigma^{3}\right)$ be the space of initial data for the polarized Gowdy spacetimes (with $C^{\infty}$ topology). There exists an open dense subset $\hat{\mathcal{P}}\left(\Sigma^{3}\right) \subset \mathcal{P}\left(\Sigma^{3}\right)$ such that the maximal development of any set of data in $\hat{\mathcal{P}}\left(\Sigma^{3}\right)$ is inextendible.

\subsection{General $T^{3}$-case}

In the general $T^{3}$-case, the result is an immediate consequence of Proposition 3, Theorem 3 and the fact that solutions are future causally-geodesically complete. Let us quote the exact statement [83, Corollary 1, p. 1190-1191]:

Theorem 8 Consider the set of smooth, periodic initial data $\mathcal{S}_{i, p, c}$ to Equations (16) - (17) satisfying Equation (50). There is a subset $\mathcal{G}_{i, c}$ of $\mathcal{S}_{i, p, c}$ with the following properties

- $\mathcal{G}_{i, c}$ is open with respect to the $C^{1} \times C^{0}$-topology on $\mathcal{S}_{i, p, c}$,

- $\mathcal{G}_{i, c}$ is dense with respect to the $C^{\infty}$-topology on $\mathcal{S}_{i, p, c}$,

- every spacetime corresponding to initial data in $\mathcal{G}_{i, c}$ has the property that in one time direction, it is causally geodesically complete, and in the opposite time direction, the Kretschmann scalar $R_{\alpha \beta \gamma \delta} R^{\alpha \beta \gamma \delta}$ is unbounded along every inextendible causal curve,

- for every spacetime corresponding to initial data in $\mathcal{G}_{i, c}$, the $M G H D$ is $C^{2}$-inextendible.

For the sake of completeness, let us also recall the definition of $C^{2}$-inextendibility.

Definition 9 Let $(M, g)$ be a connected Lorentz manifold, which is at least $C^{2}$. Assume there is a connected $C^{2}$ Lorentz manifold $(\hat{M}, \hat{g})$ of the same dimension as $M$ and an isometric embedding $i: M \rightarrow \hat{M}$ such that $i(M) \neq \hat{M}$. Then $M$ is said to be $C^{2}$-extendible. If $(M, g)$ is not $C^{2}$ extendible, it is said to be $C^{2}$-inextendible. 
It might be possible to obtain this result using different methods. In fact, let $\mathcal{G}$ be the set of initial data such that the corresponding solutions have an asymptotic velocity, which is different from one on a dense subset of the singularity. Endowing the initial data with the $C^{\infty}$-topology, it is possible to show that $\mathcal{G}$ is a dense $G_{\delta}$ set [77]. Due to its definition, it is clear that solutions corresponding to initial data in $\mathcal{G}$ have the property that the curvature blows up on a dense subset of the singularity. It would be natural to expect the corresponding solutions to be inextendible, but providing a proof is nontrivial. Important steps in the direction of proving this statement were taken in [22]. However, to the best of our knowledge, there is, as yet, no result to this effect. 


\section{Acknowledgments}

Thanks are due to Piotr Chruściel and Alan Rendall for comments on an earlier version of this review. The author acknowledges the support of the Göran Gustafsson Foundation and the Swedish Research Council. This review article was in part written during a stay by the author at the Max Planck Institute for Gravitational Physics as a recipient of a Friedrich Wilhelm Bessel Research Award granted by the Alexander von Humboldt Stiftung. The author is a Royal Swedish Academy of Sciences Research Fellow supported by a grant from the Knut and Alice Wallenberg Foundation. 


\section{References}

[1] Anderson, M., "On long-time evolution in general relativity and geometrization of 3manifolds", Commun. Math. Phys., 222, 533-567, (2001). [DOI], [gr-qc/0006042]. (Cited on page 42.)

[2] Andersson, L. and Rendall, A.D., "Quiescent cosmological singularities", Commun. Math. Phys., 218, 479-511, (2001). [DOI], [gr-qc/0001047]. (Cited on page 24.)

[3] Andersson, L., van Elst, H. and Uggla, C., "Gowdy phenomenology in scale-invariant variables", Class. Quantum Grav., 21, S29-S57, (2004). [DOI], [gr-qc/0310127]. (Cited on page 23.)

[4] Andréasson, H., "Global foliations of matter spacetimes with Gowdy symmetry", Commun. Math. Phys., 206, 337-365, (1999). [DOI], [gr-qc/9812035]. (Cited on page 14.)

[5] Andréasson, H., Rendall, A.D. and Weaver, M., "Existence of CMC and constant areal time foliations in $T^{2}$ symmetric spacetimes with Vlasov matter", Commun. Part. Diff. Eq., 29, 237-262, (2004). [DOI], [gr-qc/0211063]. (Cited on page 14.)

[6] Belinskii, V.A., Khalatnikov, I.M. and Lifshitz, E.M., "Oscillatory Approach to a Singular Point in the Relativistic Cosmology", Adv. Phys., 19, 525-573, (1970). [DOI]. (Cited on page 22.)

[7] Belinskii, V.A., Khalatnikov, I.M. and Lifshitz, E.M., "A general solution of the Einstein equations with a time singularity", Adv. Phys., 31, 639-667, (1982). [DOI]. (Cited on page 22.)

[8] Berger, B.K., "Asymptotic Behavior of a Class of Expanding Gowdy Spacetimes", arXiv eprint, (2002). [gr-qc/0207035]. (Cited on page 44.)

[9] Berger, B.K., Chruściel, P.T., Isenberg, J. and Moncrief, V., "Global Foliations of Vacuum Spacetimes with $T^{2}$ Isometry", Ann. Phys. (N.Y.), 260, 117-148, (1997). [DOI], [grqc/9702007]. (Cited on page 14.)

[10] Berger, B.K. and Garfinkle, D., "Phenomenology of the Gowdy universe on $T^{3} \times R$ ", Phys. Rev. D, 57, 4767-4777, (1998). [DOI], [gr-qc/9710102]. (Cited on pages 30 and 32.)

[11] Berger, B.K. and Moncrief, V., "Numerical Investigation of Cosmological Singularities", Phys. Rev. D, 48, 4676-4687, (1993). [DOI], [gr-qc/9307032]. (Cited on page 32.)

[12] Chae, M. and Chruściel, P.T., "On the dynamics of Gowdy space times", Commun. Pure Appl. Math., 57, 1015-1074, (2004). [DOI], [gr-qc/0305029]. (Cited on pages 37, 38, and 40.)

[13] Choquet-Bruhat, Y. and Geroch, R., "Global aspects of the Cauchy problem in General Relativity", Commun. Math. Phys., 14, 329-335, (1969). [DOI]. (Cited on page 18.)

[14] Christodoulou, D., "The instability of naked singularities in the gravitational collapse of a scalar field", Ann. Math. (2), 149, 183-217, (1999). [DOI]. (Cited on page 19.)

[15] Christodoulou, D., Mathematical Problems of General Relativity I, Zurich Lectures in Advanced Mathematics, (EMS Publishing House, Zürich, 2008). [DOI]. (Cited on page 16.)

[16] Chruściel, P.T., "On Space-Times with $U(1) \times U(1)$ Symmetric Compact Cauchy Surfaces", Ann. Phys. (N.Y.), 202, 100-150, (1990). [DOI]. (Cited on pages 11, 12, 13, and 14.)

[17] Chruściel, P.T., On Uniqueness in the Large of Solutions of Einstein's Equations (Strong Cosmic Censorship), CMA Proceedings, 27, (Australian National University Press, Canberra, 1991). (Cited on pages 10 and 19.) 
[18] Chruściel, P.T., "On completeness of orbits of Killing vector fields", Class. Quantum Grav., 10, 2091-2101, (1993). [DOI], [gr-qc/9304029]. (Cited on page 10.)

[19] Chruściel, P.T., Galloway, G. and Pollack, D., Mathematical general relativity: a sampler, Preprint Series, 03, (Institut Mittag-Leffler, Djursholm, Sweden, 2009). URL (accessed 8 January 2010):

http://www.mittag-leffler.se/preprints/0809f/info.php?id=03. (Cited on page 23.)

[20] Chruściel, P.T. and Isenberg, J., "Non-isometric vacuum extensions of vacuum maximal globally hyperbolic spacetimes", Phys. Rev. D, 48, 1616-1628, (1993). [DOI]. (Cited on pages 19, 20 , and 21.)

[21] Chruściel, P.T., Isenberg, J. and Moncrief, V., "Strong Cosmic Censorship in Polarized Gowdy Spacetimes", Class. Quantum Grav., 7, 1671-1680, (1990). [DOI]. (Cited on pages 13, 22, 28, 42 , and 51.)

[22] Chruściel, P.T. and Lake, K., "Cauchy horizons in Gowdy spacetimes", Class. Quantum Grav., 21, S153-S169, (2004). [DOI], [gr-qc/0307088]. (Cited on page 52.)

[23] Dafermos, M., "Stability and instability of the Cauchy horizon for the spherically symmetric Einstein-Maxwell-scalar field equations", Ann. Math., 158, 875-928, (2003). [DOI]. (Cited on page 19.)

[24] Dafermos, M., "The interior of charged black holes and the problem of uniqueness in general relativity", Commun. Pure Appl. Math., 58, 445-504, (2005). [DOI], [gr-qc/0307013]. (Cited on page 19.)

[25] Dafermos, M. and Rendall, A.D., "Inextendibility of expanding cosmological models with symmetry", Class. Quantum Grav., 22, L143-L147, (2005). [DOI], [gr-qc/0509106]. (Cited on page 22.)

[26] Dafermos, M. and Rendall, A.D., "Strong cosmic censorship for $T^{2}$-symmetric cosmological spacetimes with collisionless matter", arXiv e-print, (2006). [gr-qc/0610075]. (Cited on page 22.)

[27] Dafermos, M. and Rendall, A.D., "Strong cosmic censorship for surface-symmetric cosmological spacetimes with collisionless matter", arXiv e-print, (2007). [gr-qc/0701034]. (Cited on page 22.)

[28] Damour, T., Henneaux, M. and Nicolai, H., "Cosmological billiards", Class. Quantum Grav., 20, R145-R200, (2003). [DOI], [hep-th/0212256]. (Cited on page 23.)

[29] Damour, T., Henneaux, M., Rendall, A.D. and Weaver, M., "Kasner-like behaviour for subcritical Einstein-matter systems", Ann. Henri Poincare, 3, 1049-1111, (2002). [DOI], [grqc/0202069]. (Cited on page 24.)

[30] Damour, T. and Nicolai, H., "Higher order M-theory corrections and the Kac-Moody algebra $E_{10}$ ", Class. Quantum Grav., 22, 2849-2880, (2005). [DOI], [hep-th/0504153]. (Cited on page 23.)

[31] Eardley, D., Liang, E. and Sachs, R., "Velocity-Dominated Singularities in Irrotational Dust Cosmologies", J. Math. Phys., 13, 99-107, (1972). [DOI]. (Cited on page 23.)

[32] Eardley, D.M. and Moncrief, V., "The Global Existence Problem and Cosmic Censorship in General Relativity", Gen. Relativ. Gravit., 13, 887-892, (1981). [DOI]. (Cited on page 19.) 
[33] Fischer, A.E. and Moncrief, V., "The reduced Einstein equations and the conformal volume collapse of 3-manifolds", Class. Quantum Grav., 18, 4493-4515, (2001). [DOI]. (Cited on page 42.)

[34] Fourès-Bruhat, Y., "Théorème d'existence pour certains systèmes d'équations aux dérivées partielles non linéaires", Acta Math., 88, 141-225, (1952). [DOI]. (Cited on page 18.)

[35] Friedrich, H. and Rendall, A.D., "The Cauchy problem for the Einstein equations", in Schmidt, B.G., ed., Einstein's Field Equations and Their Physical Implications: Selected Essays in Honour of Jürgen Ehlers, Lecture Notes in Physics, 540, pp. 127-223, (Springer, Berlin; New York, 2000). [gr-qc/0002074], [Google Books]. (Cited on page 16.)

[36] Garfinkle, D. and Weaver, M., "High velocity spikes in Gowdy spacetimes", Phys. Rev. D, 67, 124009, (2003). [DOI], [gr-qc/0303017]. (Cited on page 36.)

[37] Gowdy, R.H., "Errata: Gravitational Waves in Closed Universes", Phys. Rev. Lett., 27, 1102, (1971). [DOI]. (Cited on page 11.)

[38] Gowdy, R.H., "Gravitational Waves in Closed Universes", Phys. Rev. Lett., 27, 826-829, (1971). [DOI]. (Cited on page 11.)

[39] Gowdy, R.H., "Vacuum Spacetimes with Two-Parameter Spacelike Isometry Groups and Compact Invariant Hypersurfaces: Topologies and Boundary Conditions", Ann. Phys. (N.Y.), 83, 203-241, (1974). [DOI]. (Cited on page 11.)

[40] Grubišić, B. and Moncrief, V., "Asymptotic behaviour of the $T^{3} \times R$ Gowdy space-times", Phys. Rev. D, 47, 2371-2382, (1993). [DOI], [gr-qc/9209006]. (Cited on pages 22, 24, and 30.)

[41] Hawking, S.W., "The Occurrence of singularities in cosmology. III. Causality and singularities", Proc. R. Soc. London, Ser. A, 300, 187-201, (1967). [ADS]. (Cited on page 20.)

[42] Hawking, S.W. and Ellis, G.F.R., The Large Scale Structure of Space-Time, Cambridge Monographs on Mathematical Physics, (Cambridge University Press, Cambridge, 1973). [Google Books]. (Cited on page 20.)

[43] Hawking, S.W. and Penrose, R., "The singularities of gravitational collapse and cosmology", Proc. R. Soc. London, Ser. A, 314, 529-548, (1970). [ADS]. (Cited on page 20.)

[44] Heinzle, J.M. and Ringström, H., "Future asymptotics of vacuum Bianchi type VI $\mathrm{I}_{0}$ solutions", Class. Quantum Grav., 26, 145001, (2009). [DOI]. (Cited on pages 11 and 23.)

[45] Heinzle, J.M. and Uggla, C., "Mixmaster: fact and belief", Class. Quantum Grav., 26, 075016, (2009). [DOI], [arXiv:0901.0776]. (Cited on page 11.)

[46] Heinzle, J.M. and Uggla, C., "A new proof of the Bianchi type IX attractor theorem", Class. Quantum Grav., 26, 075015, (2009). [DOI], [arXiv:0901.0806]. (Cited on page 11.)

[47] Heinzle, J.M., Uggla, C. and Röhr, N., "The cosmological billiard attractor", Adv. Theor. Math. Phys., 13, 293-407, (2009). [gr-qc/0702141]. (Cited on page 23.)

[48] Isenberg, J. and Kichenassamy, S., "Asymptotic behavior in polarized $T^{2}$-symmetric vacuum space-times", J. Math. Phys., 40, 340-352, (1999). [DOI]. (Cited on page 24.)

[49] Isenberg, J. and Moncrief, V., "The Existence of Constant Mean Curvature Foliations of Gowdy 3-Torus Spacetimes", Commun. Math. Phys., 86, 485-493, (1983). [DOI]. Online version (accessed 12 March 2010):

http://projecteuclid.org/euclid.cmp/1103921839. (Cited on page 14.) 
[50] Isenberg, J. and Moncrief, V., "Asymptotic behavior of the gravitational field and the nature of singularities in Gowdy spacetimes", Ann. Phys. (N.Y.), 199, 84-122, (1990). [DOI]. (Cited on pages $13,22,23,25,28$, and 29 .)

[51] Isenberg, J. and Weaver, M., "On the area of the symmetry orbits in $T^{2}$ symmetric spacetimes", Class. Quantum Grav., 20, 3783-3796, (2003). [DOI], [gr-qc/0304019]. (Cited on page 14.)

[52] Jurke, T., "On future asymptotics of polarized Gowdy $T^{3}$-models", Class. Quantum Grav., 20, 173-191, (2003). [DOI], [gr-qc/0210022]. (Cited on pages 42 and 51.)

[53] Kichenassamy, S., Nonlinear Wave Equations, Monographs and Textbooks in Pure and Applied Mathematics, 194, (Marcel Dekker, New York, 1996). [Google Books]. (Cited on page 24.)

[54] Kichenassamy, S. and Rendall, A.D., "Analytic description of singularities in Gowdy spacetimes", Class. Quantum Grav., 15, 1339-1355, (1998). [DOI]. (Cited on pages 24, 30, 31, and 40.)

[55] Lifshitz, E.M. and Khalatnikov, I.M., "Investigations in relativistic cosmology", Adv. Phys., 12, 185-249, (1963). [DOI]. (Cited on page 22.)

[56] Misner, C.W., "Mixmaster Universe", Phys. Rev. Lett., 22, 1071-1074, (1969). [DOI]. (Cited on page 23.)

[57] Misner, C.W., Thorne, K.S. and Wheeler, J.A., Gravitation, (W.H. Freeman, San Fransisco, 1973). (Cited on page 23.)

[58] Moncrief, V., "Global Properties of Gowdy Spacetimes with $T^{3} \times R$ Topology", Ann. Phys. (N.Y.), 132, 87-107, (1981). [DOI]. (Cited on page 14.)

[59] Mostert, P.S., "On a compact Lie group acting on a manifold", Ann. Math., 65, 447-455, (1957). (Cited on pages 10 and 11.)

[60] O'Neill, B., Semi-Riemannian Geometry: With Applications to Relativity, Pure and Applied Mathematics, 103, (Academic Press, San Diego; London, 1983). [Google Books]. (Cited on pages 18,20 , and 22.)

[61] Penrose, R., "Gravitational Collapse and Space-Time Singularities", Phys. Rev. Lett., 14, 57-59, (1965). [DOI]. (Cited on page 20.)

[62] Penrose, R., "Gravitational Collapse: The Role of General Relativity", Riv. Nuovo Cimento, 1, 252-276, (1969). [ADS]. (Cited on page 16.)

[63] Penrose, R., "Singularities and Time-Asymmetry", in Hawking, S.W. and Israel, W., eds., General Relativity: An Einstein Centenary Survey, 1, pp. 581-638, (Cambridge University Press, Cambridge; New York, 1979). (Cited on page 16.)

[64] Penrose, R., "Gravitational Collapse: The Role of General Relativity", Gen. Relativ. Gravit., 34, 1141-1165, (2002). [DOI]. Reprint of Riv. Nuovo Cimento, 1, 257, (1969). (Cited on page 16.)

[65] Rendall, A.D., "Reduction of the Characteristic Initial Value Problem to the Cauchy Problem and Its Applications to the Einstein Equations", Proc. R. Soc. London, Ser. A, 427, 221-239, (1990). [DOI]. (Cited on page 17.) 
[66] Rendall, A.D., "Constant mean curvature foliations in cosmological spacetimes", Helv. Phys. Acta, 69, 490-500, (1996). [gr-qc/9606049]. (Cited on page 14.)

[67] Rendall, A.D., "Existence of constant mean curvature foliations in spacetimes with twodimensional local symmetry", Commun. Math. Phys., 189, 145-164, (1997). [DOI], [grqc/9605022]. (Cited on pages 11 and 14.)

[68] Rendall, A.D., "Fuchsian analysis of singularities in Gowdy spacetimes beyond analyticity", Class. Quantum Grav., 17, 3305-3316, (2000). [DOI], [gr-qc/0004044]. (Cited on pages 24, 31, 32 , and 40.)

[69] Rendall, A.D., "Theorems on Existence and Global Dynamics for the Einstein Equations", Living Rev. Relativity, 8, lrr-2005-6, (2005). URL (accessed 7 August 2009): http://www.livingreviews.org/lrr-2005-6. (Cited on page 15.)

[70] Rendall, A.D., Partial Differential Equations in General Relativity, Oxford Graduate Texts in Mathematics, 16, (Oxford University Press, Oxford; New York, 2008). (Cited on pages 10 and 13.)

[71] Rendall, A.D. and Weaver, M., "Manufacture of Gowdy spacetimes with spikes", Class. Quantum Grav., 18, 2959-2975, (2001). [DOI], [gr-qc/0103102]. (Cited on pages 32, 36, and 40.)

[72] Ringström, H., "Curvature blow up in Bianchi VIII and IX vacuum spacetimes", Class. Quantum Grav., 4, 713-731, (2000). [DOI], [gr-qc/9911115]. (Cited on page 11.)

[73] Ringström, H., "The Bianchi IX attractor", Ann. Henri Poincare, 2, 405-500, (2001). [DOI], [gr-qc/0006035]. (Cited on page 11.)

[74] Ringström, H., "Asymptotic expansions close to the singularity in Gowdy spacetimes", Class. Quantum Grav., 21, S305-S322, (2004). [DOI], [gr-qc/0303051]. (Cited on pages 37 and 38.)

[75] Ringström, H., "On a wave map equation arising in general relativity", Commun. Pure Appl. Math., 57, 657-703, (2004). [DOI]. (Cited on pages 44, 45, 46, 47, 48, 49, and 50.)

[76] Ringström, H., "On Gowdy vacuum spacetimes", Math. Proc. Camb. Phil. Soc., 136, 485-512, (2004). [DOI], [gr-qc/0204044]. (Cited on pages 37 and 38.)

[77] Ringström, H., "Curvature blow up on a dense subset of the singularity in $T^{3}$-Gowdy", $J$. Hyperbol. Differ. Equations, 2, 547-564, (2005). [DOI]. (Cited on page 52.)

[78] Ringström, H., "Data at the moment of infinite expansion for polarized Gowdy", Class. Quantum Grav., 22, 1647-1653, (2005). [DOI]. (Cited on page 42.)

[79] Ringström, H., "Existence of an asymptotic velocity and implications for the asymptotic behaviour in the direction of the singularity in $T^{3}$-Gowdy", Commun. Pure Appl. Math., 59, 977-1041, (2006). [DOI]. (Cited on pages 37, 38, 39, 40, and 41.)

[80] Ringström, H., "On curvature decay in expanding cosmological models", Commun. Math. Phys., 264, 613-630, (2006). [DOI]. (Cited on page 42.)

[81] Ringström, H., "On the $T^{3}$-Gowdy Symmetric Einstein-Maxwell Equations", Ann. Henri Poincare, 7, 1-20, (2006). [DOI]. (Cited on page 46.)

[82] Ringström, H., The Cauchy Problem in General Relativity, ESI Lectures in Mathematics and Physics, (EMS Publishing House, Zürich, 2009). [DOI], [Google Books]. (Cited on pages 18, 19, 20, and 21.) 
[83] Ringström, H., "Strong cosmic censorship in $T^{3}$-Gowdy spacetimes", Ann. Math., 170, 11811240, (2009). (Cited on pages 38, 41, and 51.)

[84] Smulevici, J., "Strong Cosmic Censorship for $T^{2}$-Symmetric Spacetimes with Cosmological Constant and Matter", Ann. Henri Poincare, 9, 1425-1453, (2008). [DOI], [arXiv:0710.1351]. (Cited on page 22.)

[85] Smulevici, J., "On the area of the symmetry orbits of cosmological spacetimes with toroidal or hyperbolic symmetry", arXiv e-print, (2009). [arXiv:0904.0806]. (Cited on page 14.)

[86] Ståhl, F., "Fuchsian analysis of $\mathbf{S}^{2} \times \mathbf{S}^{1}$ and $\mathbf{S}^{3}$ Gowdy spacetimes", Class. Quantum Grav., 19, 4483-4504, (2002). [DOI], [gr-qc/0109011]. (Cited on pages 24 and 31.)

[87] Tanimoto, M., "Locally $U(1) \times U(1)$ symmetric cosmological models", Class. Quantum Grav., 18, 479-507, (2001). [DOI], [gr-qc/0003033]. (Cited on page 11.)

[88] Uggla, C., van Elst, H., Wainwright, J. and Ellis, G.F.R., "The past attractor in inhomogeneous cosmology", Phys. Rev. D, 68, 103502, (2003). [DOI], [gr-qc/0304002]. (Cited on page 23.)

[89] Wald, R.M., General Relativity, (University of Chicago Press, Chicago, 1984). (Cited on page 20.)

[90] Wald, R.M., "Gravitational Collapse and Cosmic Censorship", arXiv e-print, (1997). [grqc/9710068]. (Cited on pages 16 and 19.)

[91] Weaver, M., "On the area of the symmetry orbits in $T^{2}$ symmetric pacetimes with Vlasov matter", Class. Quantum Grav., 21, 1079-1097, (2004). [DOI], [gr-qc/0308055]. (Cited on page 14.) 